PREPARED FOR THE U.S. DEPARTMENT OF ENERGY, UNDER CONTRACT DE-AC02-76CH03073

PPPL-3706

PPPL-3706

UC-70

Physical Limitations of Empirical Field Models:

Force Balance and Plasma Pressure

by

Sorin Zaharia and C.Z. Cheng

June 2002

NM|

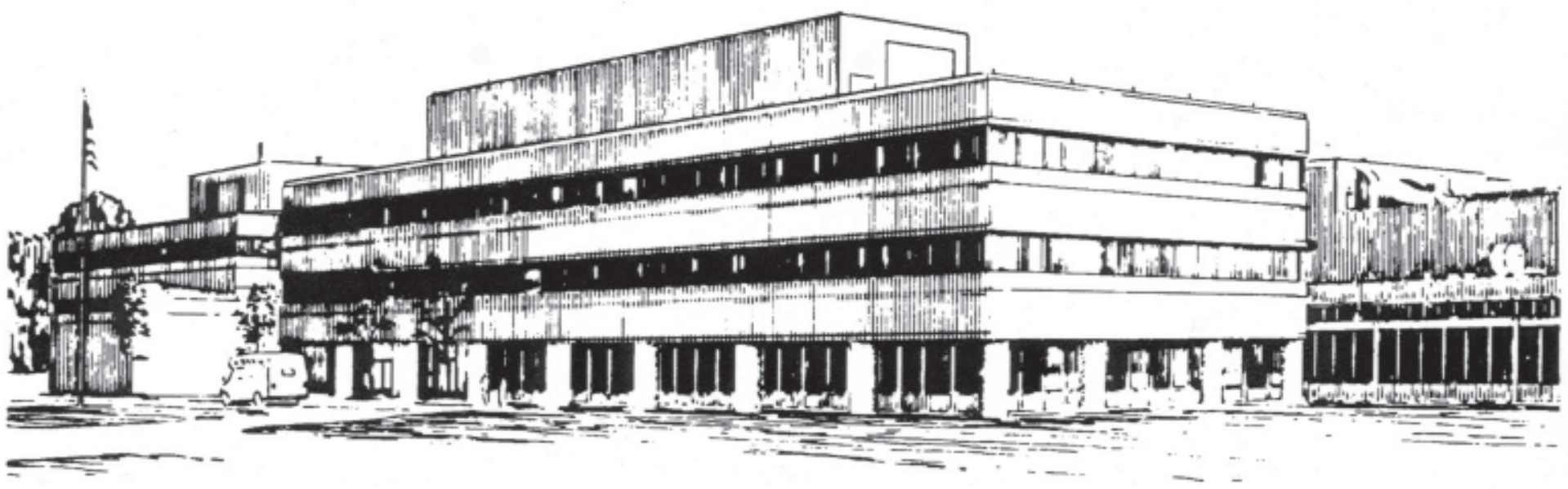

PRINCETON PLASMA PHYSICS LABORATORY PRINCETON UNIVERSITY, PRINCETON, NEW JERSEY 


\section{PPPL Reports Disclaimer}

This report was prepared as an account of work sponsored by an agency of the United States Government. Neither the United States Government nor any agency thereof, nor any of their employees, makes any warranty, express or implied, or assumes any legal liability or responsibility for the accuracy, completeness, or usefulness of any information, apparatus, product, or process disclosed, or represents that its use would not infringe privately owned rights. Reference herein to any specific commercial product, process, or service by trade name, trademark, manufacturer, or otherwise, does not necessarily constitute or imply its endorsement, recommendation, or favoring by the United States Government or any agency thereof. The views and opinions of authors expressed herein do not necessarily state or reflect those of the United States Government or any agency thereof.

\section{Availability}

This report is posted on the U.S. Department of Energy's Princeton Plasma Physics Laboratory Publications and Reports web site in Fiscal Year 2002. The home page for PPPL Reports and Publications is: http://www.pppl.gov/pub_report/

DOE and DOE Contractors can obtain copies of this report from:

U.S. Department of Energy

Office of Scientific and Technical Information

DOE Technical Information Services (DTIS)

P.O. Box 62

Oak Ridge, TN 37831

Telephone: (865) 576-8401

Fax: (865) 576-5728

Email: reports@adonis.osti.gov

This report is available to the general public from:

National Technical Information Service

U.S. Department of Commerce

5285 Port Royal Road

Springfield, VA 22161

Telephone: 1-800-553-6847 or

(703) 605-6000

Fax: (703) 321-8547

Internet: http://www.ntis.gov/ordering.htm 


\title{
Physical limitations of empirical field models: force balance and plasma pressure
}

\author{
Sorin Zaharia and C. Z. Cheng \\ Plasma Physics Laboratory, Princeton University, Princeton, NJ 08543
}

\begin{abstract}
In this paper we study whether the magnetic field of the T96 empirical model can be in force balance with an isotropic plasma pressure distribution. Using the field of T96, we obtain values for the pressure $P$ by solving a Poisson-type equation $\nabla^{2} P=\nabla \cdot(\mathbf{J} \times \mathbf{B})$ in the equatorial plane, and 1-D profiles on the Sun-Earth axis by integrating $\boldsymbol{\nabla} P=\mathbf{J} \times \mathbf{B}$. We work in a flux coordinate system in which the magnetic field is expressed in terms of Euler potentials. Our results lead to the conclusion that the T96 model field cannot be in equilibrium with an isotropic pressure. We also analyze in detail the computation of Birkeland currents using Vasyliunas relation and the T96 field, which yields unphysical results, again indicating the lack of force balance in the empirical model. The underlying reason for the force imbalance is likely the fact that the derivatives of the least-square fitted model $\mathbf{B}$ are not accurate predictions of the actual magnetospheric field derivatives. Finally, we discuss a possible solution to the problem of lack of force balance in empirical field models.
\end{abstract}

\section{Introduction}

The goal of this work is to evaluate the T96 empirical model on physical grounds in the closed-field region of the inner and middle magnetosphere, by analyzing its limitations and applicability to tasks in which a force balance between field and plasma pressure forces is essential.

Several empirical data-based models have been developed [Olson and Pfitzer, 1974; Mead and Fairfield, 1975; Alexeev et al., 1996; Ostapenko and Maltsev, 1997; Tsyganenko, 1987, 1989, 1995; Tsyganenko and Stern, 1996]. These models are calibrated against field databases obtained from different observations and at different times, and thus the model $\mathbf{B}$ represents a large-scale time-average picture of the magnetospheric field. Common uses of empirical models include mapping along the field lines, useful for interpreting spacecraft data, and calculating the trajectory of particles in the $\mathbf{B}$ field, essential in space weather modeling.

However, for many other purposes, it is useful to also have information about the plasma, in addition to the magnetic field. A quantity that contains significant information about the plasma is the pressure, a key parameter that determines both transverse and field-aligned magnetospheric currents. The region 1 and region 2 field-aligned (Birkeland) currents in particular are crucial in regulating the magnetosphereionosphere (M-I) coupling. Even though they were discovered a while ago [Iijima and Potemra, 1976], the mechanisms responsible for their formation are still being debated, however it has become quite clear that pressure gradients play a crucial role [e.g., Cheng, 1995; Antonova and Ganyushkina, 1996]. Besides their role in M-I coupling, the Birkeland currents also affect the magnetospheric field. It has been demonstrated [e.g., Donovan, 1993] that the inclusion of $J_{\|}$ effects is essential for mapping along $\mathbf{B}$, as the field line topology is essentially affected in their presence [Ding et al., 1994].

Because a significant body of magnetospheric phenomena depend on pressure profiles, the need for a clear picture of the pressure distribution is then obvious. Unfortunately, the pressure $P$ is not well known through much of the magnetosphere, most insitu observations being either in the ionosphere or in the plasma sheet, and consequently other means are needed to obtain it. A method employed by [Wing 
and Newell, 1998] consists in mapping the ionospheric $P$ into the magnetosphere along the field lines of an empirical model. This method fits in the common practice of mapping along an empirical field, from auroral structures [Elphinstone et al., 1991; Pulkkinen et al., 1995; Ober et al., 2000] to electric potentials [e.g., Boonsiriseth et al., 2001] to field-aligned currents [e.g., Lu et al., 1997]. However, it needs to be recognized that using a non self-consistent state obtained from such a mapping as starting point for physical calculations such as the computation of Birkeland currents [e.g., Wing and Newell, 2000] is physically unsound and can even lead to spurious results, as will be shown later.

Another way of obtaining the pressure is computing a $P$ profile that would be in force balance with the magnetic stresses of an empirical field model. Before taking up such an analysis, it is natural to ask "under what conditions is the assumption of magnetospheric force balance valid ?" Force balance is thought to be valid in the so-called "quasi-equilibrium" magnetospheric states, which are believed to exist at most times, except for periods of explosive activity [e.g., Tsyganenko, 1990]. Furthermore, if the time scale of global $\mathbf{B}$ changes is not too short, one can describe the evolution of the magnetosphere as a series of still "snapshots", each of them being a unique quasi-equilibrium state. For example, studies [e.g., Kropotkin and Lui, 1995] indicate that the substorm growth phase can be described by such a sequence of quasi-equilibria.

In a quasi-equilibrium magnetospheric state, the magnetic forces should be balanced by one or a combination of: (a) a pressure gradient force, (b) a time variation of the plasma flow momentum, and/or (c) a gradient of the plasma flow kinetic energy. Of these possibilities, (b) can be excluded when analyzing an empirical model configuration, since as mentioned such a model represents an average field, in which small time-scale variations are smoothed out; (c) also is ruled out for a "quasi-static" equilibrium, in which there are no significant flows. The quasi-static condition is expected to hold best in a large region of the inner and middle magnetosphere, generally on closed field lines where only sub-Alfvenic flows exist [Wolf, 1983].

With the assumption of quasi-static equilibrium, the question is now whether the pressure is isotropic or anisotropic. We note that in-situ observations [e.g., Stiles et al., 1978; Baumjohann and Paschmann, 1989; Nakamura et al., 1991] show that during quiet times the plasma sheet is almost isotropic. Furthermore, observations of ionospheric particle fluxes [Sergeev and Malkov, 1988] revealed the existence of a sharp boundary, called the isotropy boundary, separating the pole-ward zone of isotropic precipitation from the equator-ward zone of smaller precipitating flux. Regions pole-ward of the isotropy boundary roughly map to the plasma sheet, and the isotropy is believed to be due to current-sheet scattering [Sergeev et al., 1993] because of large ion gyroradius and small field curvature in the tail. The observed isotropy is theoretically expected, as an anisotropic plasma would be highly unstable due to either of the fire hose or mirror large-scale MHD instabilities. For anisotropy values exceeding certain thresholds, these instabilities would quickly relax the plasma to an isotropic state [Nötzel et al., 1985]. The existence of these two "macro"-instabilities places very tight constraints on the possible degree of anisotropy [Walker and Southwood, 1982; Voigt, 1986; Hill and Voigt, 1992], with Hill and Voigt [1992] concluding that the maximum degree of anisotropy supportable in the plasma sheet is given by $P_{\perp} / P_{\|}$in the range $1 \pm \delta^{2}$, with $\delta \approx 0.1$. Besides the two macroinstabilities, there are additional isotropization mechanisms, such as the ion cyclotron instability, isotropizing the plasma via pitch-angle scattering, and the stochasticity of particle orbits in thin current sheets.

The above discussion points to the fact that it is realistic to look for an isotropic $P$ in equilibrium with the field of an empirical model. While there is, to our knowledge, no study of $P$ in force balance with the empirical model of Tsyganenko [1995] and Tsyganenko and Stern [1996] (from here on T96), such a task has been attempted for earlier models of Tsyganenko [1987] and Tsyganenko [1989] (from here on T87 and T89, respectively). The studies by Spence et al. [1987], Kan et al. [1992] and Lui et al. [1994] obtained isotropic or anisotropic pressure profiles in force equilibrium with magnetic stresses along the midnight meridian line in the equatorial plane. Extending the domain of calculation, Horton et al. [1993] obtained 2-D isotropic $P$ from T87 and T89 on the equatorial and noon-midnight meridional planes. They also computed an anisotropic profile in 2-D force balance with an analytical approximation of the empirical B. A similar but more complete analysis in 2-D also, without resorting to the approximations of Horton et al. [1993], was performed by Cao and Lee [1994] for T87 and T89 on the same two planes. While they found T87 to be "almost isotropic", the signif- 
icant anisotropy found in the T89 case is likely an artifact due to the fact that T89 is over-stretched in the tail region [e.g. Peredo et al., 1993; Rostoker and Skone, 1993]. This being said, we note that very close to Earth significant anisotropy might indeed exist, as observations [Lui and Hamilton, 1992] indicate. However, $P$ is less important in regions with low plasma $\beta=2 P / B^{2}$, because in such regions $P$ does not alter the magnetic configuration much [e.g., Cheng, 1992]. This is certainly the case for example at $4.5 \mathrm{R}_{\mathrm{E}}$, where $C a o$ and Lee [1994] find a rather large anisotropy $\left(P_{\perp} / P_{p}\right.$ arallel $\left.=0.3\right)$, but where plasma $\beta$ is very low, as observations show [e.g., Lui and Hamilton, 1992].

Our study of the T96 model is motivated by many factors. First, as opposed to earlier models, which were parameterized by the $K_{p}$ index and had no fieldaligned currents, the inclusion of those currents and the parameterization of T96 on solar wind conditions should result in a more accurate rendition of the global magnetospheric picture. Secondly, the studies of Cao and Lee [1994] and Horton et al. [1993] investigate a limited domain. The former is limited to $15 R_{E}$, thus not including the important high- $\beta$ plasma sheet region down the tail. On the other hand, Horton et al. [1993] only consider distances farther than $10 R_{E}$, thereby missing the crucial transitional region between $6.6 R_{E}$ and $10 R_{E}$, where the $\mathbf{B}$ field transforms from a dipole-like to an extended tail-like configuration and plasma $\beta$ starts to be significant. Third, none of the two studies look at the day-side magnetosphere, where nevertheless observations exist [Lui and Hamilton, 1992]. Finally, earlier 2-D investigations [Horton et al., 1993] presented $P$ distributions obtained by 2-D quadratures of $\mathbf{J} \times \mathbf{B}$, which is not a well-posed problem. Our more rigorous approach consists in de-composing $\mathbf{J} \times \mathbf{B}$ into a curl-free part and a divergence-free part, followed by computation of $P$ from a Poisson equation.

We work in a flux coordinate system [e.g., Cheng, $1992,1995]$, in which the empirical $\mathbf{B}$ is expressed in terms of Euler potentials [e.g., Stern, 1994a]. This magnetic field representation, described in Section 2, provides clear physical meaning to formulas expressing various magnetospheric quantities. In Section 3 we present our methods for obtaining $P$ : both a direct integration of $\mathbf{J} \times \mathbf{B}$ from the empirical model in 1-D on the Sun-Earth line and a 2-D solution of $\nabla^{2} P=\boldsymbol{\nabla} \cdot(\mathbf{J} \times \mathbf{B})$ in the equatorial plane. In Section 4 we briefly describe our numerical method and how the computational domain is defined. The re- sults for $P$ and their discussion are given in Section 5. In Section 6, we show that the Birkeland currents as computed by using Vasyliunas relation [Vasyliunas, 1970, 1984] and the T96 field can be unrealistic. Section 7 describes a possible solution to the force balance problem in empirical models, and a summary and conclusions are given in Section 8 .

The main result of our study is that the T96 empirical model is not in force balance in 3-D with any isotropic pressure distribution. A possible reason for this, despite the ability of the model to describe relatively well the magnetospheric $\mathbf{B}$, lies in the method by which the model is built, which is by fitting the field from a framework of postulated currents to observed $\mathbf{B}$ data. This approach, while providing $\mathbf{B}$ that correlates well with observations, could yield less than satisfactory values for key physical quantities computed from the model by differentiation of the field Stern [1994a].

\section{Physical Quantities in a Magneto- spheric Flux Coordinate System}

The equation of motion for plasma in a single-fluid approach, with isotropic $P$ and over time scales long enough such that the quasi-neutrality condition is satisfied is, in rationalized EMU units,

$$
\rho\left[\frac{\partial \mathbf{v}}{\partial t}+(\mathbf{v} \cdot \nabla) \mathbf{v}\right]=\mathbf{J} \times \mathbf{B}-\nabla P
$$

A quasi-static equilibrium exists (left-hand side of Eq. 1 negligible) if: (a) there is no time-dependence on the time scale of interest $(\partial / \partial t \approx 0)$, and $(b)$, the convective term $(\mathbf{v} \cdot \boldsymbol{\nabla}) \mathbf{v}$ is negligible. In such a case, the equations describing our system are:

$$
\begin{gathered}
\mathbf{J} \times \mathbf{B}=\nabla P \\
\boldsymbol{\nabla} \times \mathbf{B}=\mathbf{J} \\
\boldsymbol{\nabla} \cdot \mathbf{B}=0
\end{gathered}
$$

\subsection{Euler potential representation of $B$; flux coordinate system}

From Eq. $4 \mathbf{B}$ can be expressed as

$$
\mathbf{B}=\nabla \psi \times \nabla \alpha,
$$


where $\psi$ and $\alpha$ are called Euler potentials [e.g., Stern, 1967]. Obviously $\mathbf{B} \cdot \boldsymbol{\nabla} \psi=\mathbf{B} \cdot \boldsymbol{\nabla} \alpha=0$, so that the magnetic field lines are defined by the intersection between constant $\psi$ and constant $\alpha$ surfaces.

The Euler potentials $\psi$ and $\alpha$ can be obtained in analytical form only for certain simplified cases, such as a dipole field [Stern, 1976] or certain force-free fields [e.g., Stern, 1994b]. In the magnetospheric case however, the complexity of $\mathbf{B}$ precludes a simple analytical expression for $\psi$ and $\alpha$.

One approach then is computing $\psi$ and $\alpha$ numerically, and encouraging work has been reported [e.g., Cheng, 1995; Peymirat and Fontaine, 1999]. Our method, also used in [Cheng, 1995], relies on the close connection in the magnetospheric case between the Euler potential representation and the socalled "straight field line" coordinate system (see [e.g., D'haeseleer et al., 1991] and references therein). It also provides unambiguous values for $\psi$ and $\alpha$ due to physical constraints. Since our particular representation is not very common in the space physics community, it is worthwhile to briefly discuss how our flux coordinate system is built. By definition, flux coordinates are curvilinear coordinates in which the equation of a magnetic field line takes the form of a straight line [D'haeseleer et al., 1991]. For this reason these coordinates are also called straight-B or straight-field coordinates.

In the magnetosphere, the magnetic field lines form a set of "nested" surfaces ( $L$-shells), each surface being defined by field lines with footpoints at the same magnetic latitude on the Earth's surface. Let these surfaces, called magnetic (or flux) surfaces, be "labeled" by a function $\rho$, with its value constant on a surface. Let also the poloidal and toroidal (azimuthal) angles $\theta$ and $\phi$, respectively, specify a point on the surface. We have to mention that the "nestedness" constraint is satisfied in the absence of singular structures such as X-lines, and in the magnetospheric case it will be valid if reconnection regions are not included.

Since $\mathbf{B} \cdot \boldsymbol{\nabla} \rho=0, \mathbf{B}$ can be expressed in its contravariant form as $\mathbf{B}=B^{\theta} \mathbf{e}_{\theta}+B^{\phi} \mathbf{e}_{\phi}$, where $\mathbf{e}_{\theta}$ and $\mathbf{e}_{\phi}$ represent the unit vectors in the $\theta$ and $\phi$ directions, respectively, part of the co-variant basis $\left\{\mathbf{e}_{\rho}, \mathbf{e}_{\phi}, \mathbf{e}_{\theta}\right\}$. Considering the contra-variant basis $\{\boldsymbol{\nabla} \rho, \boldsymbol{\nabla} \phi, \boldsymbol{\nabla} \theta\}$ now, we have $B^{\theta}=\mathbf{B} \cdot \boldsymbol{\nabla} \theta, B^{\phi}=\mathbf{B} \cdot \boldsymbol{\nabla} \phi$ and $B^{\rho}=$ $\mathbf{B} \cdot \boldsymbol{\nabla} \rho=0$, and thus the equation for a field line is $d \theta / d \phi=B^{\theta} / B^{\phi}=(\mathbf{B} \cdot \boldsymbol{\nabla} \theta) /(\mathbf{B} \cdot \boldsymbol{\nabla} \phi)$. Generally the ratio $B^{\theta} / B^{\phi}$ is not constant on a flux surface, but it is possible [e.g., D'haeseleer et al., 1991] to make a transformation from the real angles $(\theta, \phi)$ to two variables $(\chi, \zeta)$ such that the field line is a straight line in $(\chi, \zeta): B^{\chi} / B^{\zeta}=f(\rho)$. Such a transformation is not unique, and one possibility is $\chi=\theta$ and $\zeta=$ $\phi-2 \pi \tilde{\nu} / \dot{\Psi}_{\text {pol }}$, with $\tilde{\nu}$ a function periodic in $\phi$ and $\theta$, and $\dot{\Psi}_{\text {pol }}$ the derivative with respect to $\rho$ of the poloidal flux contained within the surface $\rho$,

$$
\dot{\Psi}_{p o l}=\frac{d \Psi_{p o l}}{d \rho}=\frac{1}{2 \pi} \int_{0}^{2 \pi} \int_{0}^{2 \pi} d \phi d \theta J \mathbf{B} \cdot \nabla \theta
$$

With the described transformation, $\mathbf{B}$ is given by [D'haeseleer et al., 1991]:

$$
\mathbf{B}=\nabla \rho \times \nabla\left(-\frac{1}{2 \pi} \dot{\Psi}_{t o r} \chi+\frac{1}{2 \pi} \dot{\Psi}_{p o l} \zeta\right),
$$

a straight-line in $(\chi, \zeta)$. In the above equation $\dot{\Psi}_{\text {tor }}$ is the derivative with respect to $\rho$ of the toroidal flux contained within the surface $\rho$ :

$$
\dot{\Psi}_{t o r}=\frac{d \Psi_{t o r}}{d \rho}=\frac{1}{2 \pi} \int_{0}^{2 \pi} \int_{0}^{2 \pi} d \phi d \theta J \mathbf{B} \cdot \nabla \phi
$$

and $J$ is the Jacobian of the $(\rho, \phi, \theta)$ system: $J=$ $[(\nabla \rho \times \nabla \phi) \cdot \nabla \theta]^{-1}$. In the magnetospheric case Eq. (6) further simplifies, due to the fact that $\dot{\Psi}_{t o r}=0$. This is so because the total toroidal flux within a flux surface $\Psi_{\text {tor }}=1 /(2 \pi) \iiint d^{3} x \mathbf{B} \cdot \boldsymbol{\nabla} \phi=0$, due to the fact that there is no net poloidal current into the system (the field-aligned currents cancel on average) [Cheng, 1995]. Therefore, Eq. (6) for the magnetospheric field becomes $\mathbf{B}=\boldsymbol{\nabla} \rho \times \nabla\left(\dot{\Psi}_{\text {pol }} \zeta / 2 \pi\right)$. Since $\dot{\Psi}_{\text {pol }}$ is a function of $\rho$ only, and because $\rho$ can be any function of the flux surface, with the choice $\psi \equiv \rho \equiv \Psi_{\text {pol }} /(2 \pi)$ and $\alpha \equiv \zeta$ one finally has $\mathbf{B}=\nabla \psi \times \nabla \alpha$.

We have thus obtained B in the Euler-potential form (Eq. 5), with clear physical significance for the functions $\psi$ and $\alpha: \psi$ labels a flux surface and its value is related to the poloidal flux within that flux surface, while $\alpha$ is the equivalent of the azimuthal angle.

Fig. 1 shows several constant flux surfaces in the noon-midnight meridian and equatorial planes, as well as constant $\alpha$ lines in the equatorial plane of a quiet-time T96 configuration. The functions $\psi$ and $\alpha$, together with a third coordinate $\chi$ represent a straight-B system: B has no component in the $\boldsymbol{\nabla} \psi$ or $\boldsymbol{\nabla} \zeta$ directions, and is thus a straight line parallel to 


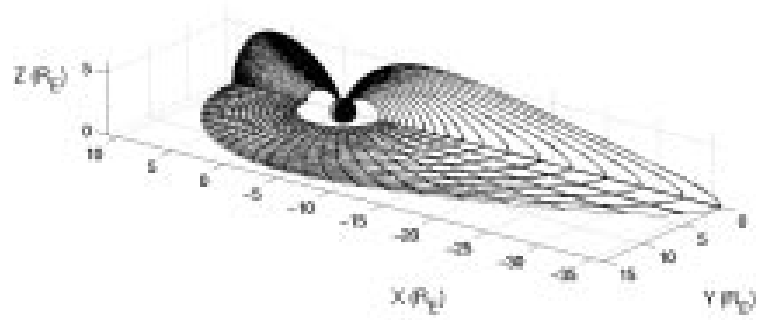

Figure 1. Constant $\psi$ surfaces in the noon-midnight meridian and equatorial planes as obtained from the T96 model with quiet-time parameters. Constant $\alpha$ lines are also shown in the equatorial plane.

$\nabla \chi$ in the $(\psi, \alpha, \chi)$ flux coordinate system. We note that this coordinate system is not orthogonal in general: $\boldsymbol{\nabla} \psi \cdot \nabla \chi \neq 0, \boldsymbol{\nabla} \psi \cdot \nabla \alpha \neq 0$, and $\boldsymbol{\nabla} \alpha \cdot \nabla \chi \neq 0$ [e.g., Cheng, 1995]. Obviously, in an axisymmetric case $\mathbf{B} \cdot \boldsymbol{\nabla} \phi=0$, so no transformation of the azimuthal angle $\phi$ would be necessary: $\alpha=\phi$.

In the general case, we note that knowing $\psi$ and $\alpha$ still leaves one degree of freedom, i.e. the choice of $\chi$, which determines the position on the field line. This choice is actually equivalent to choosing a specific form for the Jacobian of the $(\psi, \alpha, \chi)$ system, $\mathcal{J}=[(\boldsymbol{\nabla} \psi \times \nabla \zeta) \cdot \nabla \chi]^{-1}[$ e.g., Cheng, 1992, 1995]. It also has certain practical significance - for numerical calculations the choice of $\chi$ determines the spacing of grid points in the discrete computational mesh. In this paper we will make the "equal arc length" choice [Cheng, 1995], such that equal $d \chi$ correspond to equal $d s$ variations, $d s$ being the infinitesimal distance along a field line.

\subsection{Field-aligned currents}

Attempts have been made in the past [Ding et al., 1994] to obtain the Birkeland currents directly (by $J_{\|}=\mathbf{b} \cdot(\boldsymbol{\nabla} \times \mathbf{B})$, with $\left.\mathbf{b}=\mathbf{B} / B\right)$ from $\mathrm{T} 87$ or T89. Those earlier models do not have explicit fieldaligned currents built-in (unlike T96), but it appeared that because the models were built by least square fitting against large field databases, one would still find a "residual" presence of these currents, due to their existence in the actual data. However, [Ding et al., 1994] concluded that T87 and T89 do not have any such "residual" currents. They explained this apparent paradox by the fact that the magnetic field due to these currents was likely to be lost in the statistical averaging process, as they tend to flow in thin current sheets that are highly variable in both space and time [Donovan, 1993].

In a quasi-equilibrium state with isotropic $P, J_{\|}$ can be obtained from the charge neutrality condition $\boldsymbol{\nabla} \cdot \mathbf{J}=0$ by the well-known Vasyliunas relation [ $\mathrm{Va}$ syliunas, 1970, 1984]:

$$
\mathbf{B} \cdot \boldsymbol{\nabla}\left(\frac{J_{\|}}{B}\right)=\frac{2 \mathbf{B} \cdot(\boldsymbol{\nabla} P \times \boldsymbol{\kappa})}{B^{2}}=\frac{\nabla B^{2} \times \mathbf{B} \cdot \nabla P}{B^{4}},
$$

where $\boldsymbol{\kappa}=(\mathbf{b} \cdot \boldsymbol{\nabla}) \mathbf{b}$ is the magnetic field curvature. Thus, in a force-balanced state with known $P, J_{\|}$can be obtained by an integration along the line. The computation of $J_{\|}$using Eq. 7 was performed for example by Cheng [1995], using analytical $P$ profiles and a computed 3-D equilibrium field. The reverse problem has also been considered by Antonova and Ganyushkina [1996], who computed $\nabla P$ on top of the ionosphere from $J_{\|}$and using empirical model fields.

We now express Eq. 7 in our $(\psi, \alpha, \chi)$ system. First, from $\mathbf{J} \times \mathbf{B}=\boldsymbol{\nabla} P$ one has $\mathbf{B} \cdot \boldsymbol{\nabla} P=0$, and so $P$ is constant along the field line, $P=P(\psi, \alpha)$. Therefore $\boldsymbol{\nabla} P=(\partial P / \partial \alpha) \boldsymbol{\nabla} \alpha+(\partial P / \partial \psi) \boldsymbol{\nabla} \psi$, and the first equality in Eq. 7 becomes

$$
\begin{aligned}
& \mathbf{B} \cdot \boldsymbol{\nabla}\left(\frac{J_{\|}}{B}\right)=\frac{2 \mathbf{B}}{B^{2}} \cdot {\left[\frac{\partial P}{\partial \psi}(\nabla \psi \times \boldsymbol{\kappa})\right] } \\
&+\frac{2 \mathbf{B}}{B^{2}} \cdot\left[\frac{\partial P}{\partial \alpha}(\boldsymbol{\nabla} \alpha \times \boldsymbol{\kappa})\right]
\end{aligned}
$$

The equation above, however with only the first term on the right hand side (due to assuming $P=$ $P(\psi)$ only), was used by Cheng [1995]. The second term can indeed be neglected if one restricts the domain close to Earth, however past $10 R_{\mathrm{E}}$ both terms are relevant and should be included.

Each term in Eq. 8 has its own physical significance. The first term is non-zero only if the magnetic field line is not a geodesic (a curve of shortest path between two points) on the flux surface. The curvature $\boldsymbol{\kappa}$ of a geodesic line is parallel to the normal to the surface [e.g., Weinstock, 1974], i.e. to $\nabla \psi$, therefore if the field line is a geodesic then $\boldsymbol{\nabla} \psi \times \boldsymbol{\kappa}=0$ and the first term on the RHS of Eq. 8 is zero. If the line is not a geodesic however, there is a component of $\boldsymbol{\kappa}$ in the direction $\mathbf{B} \times \boldsymbol{\nabla} \psi$, called geodesic curvature, which makes the first term in Eq. 8 non-zero. In the T96 model, one can intuitively picture that due to the built-in field-aligned currents, a field line will depart from a geodesic curve. This is why we expect 
the first term in Eq. 8 to be significant if T96 is considered vs. when a model without Birkeland currents is used. The second term in Eq. 8, as opposed to the first term, is finite even with no geodesic curvature, as long as $\partial P / \partial \alpha$ is finite. We expect the regions close to the equatorial plane in the magnetotail to provide the largest contribution to this term, because $\boldsymbol{\kappa}$ is largest there, due to the stretching of the field. In the special case of axisymmetry, both terms on the RHS of Eq. 8 are zero: the first term because the $\boldsymbol{\kappa}$ is in the radial $\boldsymbol{\nabla} \psi$ direction in that case, and the second one because $\partial P / \partial \alpha \equiv \partial P / \partial \phi=0$.

\subsection{Guiding center motion; implications for the pressure distribution}

In the 3-D magnetospheric case we will show that generally $P$ depends on both $\alpha$ and $\psi$. Let us consider the bounce-averaged drift of the particle guiding center [e.g., Northrop, 1963]. We express the component of this drift in the $\nabla \psi$ direction, i.e. across flux surfaces: $\left\langle\mathbf{v}_{g c} \cdot \boldsymbol{\nabla} \psi\right\rangle=(m / q) \partial J_{2} / \partial \alpha$, where the angle brackets denote averaging over a bounce period: $\left\langle\mathbf{v}_{g c} \cdot \boldsymbol{\nabla} \psi\right\rangle=\oint\left(\mathbf{v}_{g c} \cdot \nabla \psi\right) d s / v_{\|}$, and $J_{2}=\oint v_{\|} d s$ is the second adiabatic invariant, characterizing the bounce motion of the particle between the northern and southern mirroring points.

The invariant $J_{2}$ is a function of field line: $J_{2}=$ $J_{2}(\psi, \alpha)$ [Northrop, 1963]. Considering the $\mathbf{E} \times \mathbf{B}$ convection to be weak, i.e. neglecting the electric potential energy $q \phi$ in $v_{\|}=[2(W-\mu B-q \phi) / m]^{1 / 2}$, we have

$$
\frac{\partial J_{2}}{\partial \alpha}=\frac{\partial \oint v_{\|} d s}{\partial \alpha}=\oint \frac{\partial v_{\|}}{\partial \alpha} d s=-\oint \frac{\mu}{m} \frac{\partial B}{\partial \alpha} \frac{d s}{v_{\|}},
$$

where $\mu=m v_{\perp}^{2} /(2 B)$ denotes the magnetic moment, while $W$ is the total energy of the particle. With $d \psi / d t=\mathbf{v}_{g c} \cdot \nabla \psi$ we finally obtain

$$
\left\langle\frac{d \psi}{d t}\right\rangle=-\oint \frac{\mu}{m} \frac{\partial B}{\partial \alpha} \frac{d s}{v_{\|}}
$$

which shows the rate at which the guiding center drift motion crosses the flux surfaces. Eq. 9 shows that if $\partial B / \partial \alpha=0$, the guiding center drift has no component across the flux surface. This means that in a thin shell (on the order of the ion gyro-radius) enveloping the magnetic flux surface the particle distribution would be constant as the particles $\nabla B$ and curvature-drift around the Earth, i.e. $P$ would only be a function of the flux surface: $P=P(\psi)$

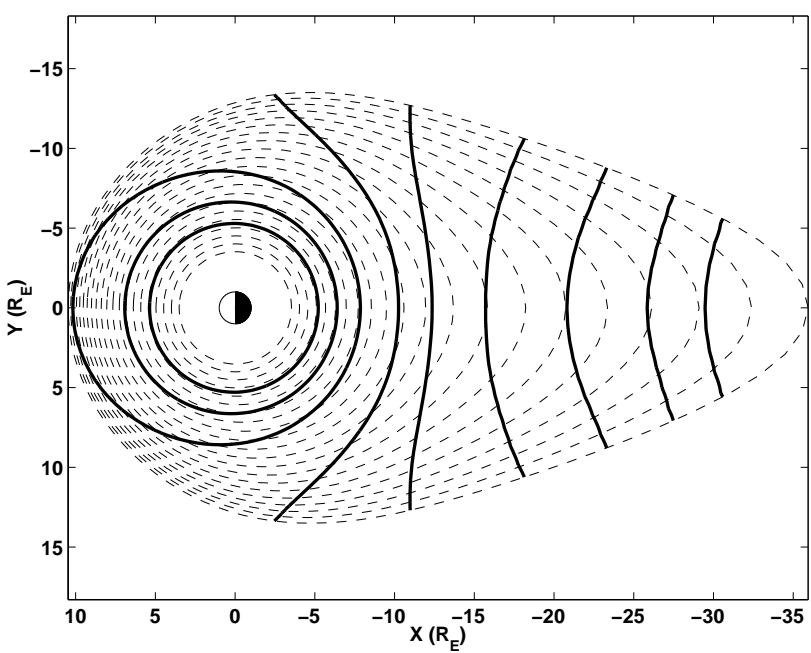

Figure 2. Constant $\psi$ (dotted lines) and B (solid lines) contours on the equatorial plane in quiet time T96; contour values for $\mathrm{B}$, from the farthest to the closest to Earth, are: 1.5, 2, 3, 5, 10, 20, 50, 100 and $200 \mathrm{nT}$

if $\partial B / \partial \alpha=0$. Generally, the systems in which the drifts lie within the magnetic surfaces are called omnigenous systems [Hall and McNamara, 1975].

The Earth's magnetosphere however does not satisfy the omnigenity constraint, as the condition $\partial B / \partial \alpha=$ 0 is not satisfied. This is seen in Fig. 2, where we plot an equatorial plane view of flux surfaces $\psi$ as well as contours of constant $|\mathbf{B}|$, as obtained from T96 with quiet-time parameters. It is apparent that while near Earth (where the field is almost dipolar) the equatorial $|\mathbf{B}|$ contours are almost circular and almost coincide with the $\psi$ contours, at distances larger than $7 \mathrm{R}_{\mathrm{E}}$ the constant $|\mathbf{B}|$ contours diverge greatly from the $\psi$ contours in the magnetotail. We note here that the addition of $\mathbf{E} \times \mathbf{B}$ convection will strengthen our conclusion that the magnetospheric $P$ has to depend on both $\psi$ and $\alpha$.

\section{Obtaining Pressure in Force Balance With a Magnetic Field Structure}

The magnetic force $\mathbf{J} \times \mathbf{B}$ can be written in a general form by using Helmholtz's decomposition theorem [e.g. Arfken, 1985]:

$$
\mathbf{J} \times \mathbf{B}=\nabla P+\nabla \times \mathbf{G}
$$




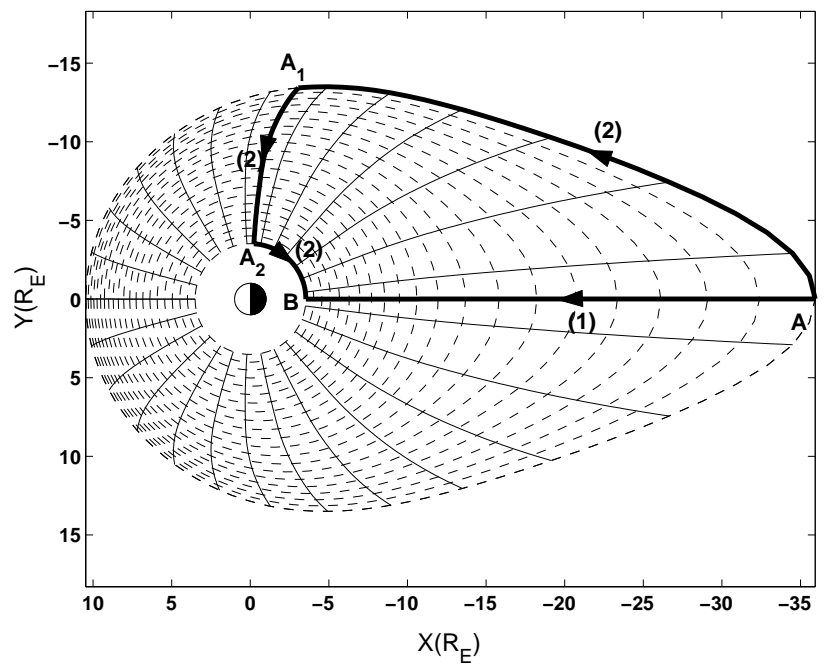

Figure 3. Two paths of integration for Eqs. 11 and 12 in the equatorial plane of T96. Several constant $\psi$ contours (dotted lines) and $\alpha$ lines (thin solid lines) are also shown.

In Eq. $10 P$ and $\mathbf{G}$ are scalar and vectorial functions of space, respectively. In the case of an exact equilibrium with isotropic pressure, one has $\boldsymbol{\nabla} \times \mathbf{G}=0$. Note that any magnetic configuration will allow the general decomposition in Eq. 10, as long as $\boldsymbol{\nabla} \cdot(\mathbf{J} \times \mathbf{B})$ and $\boldsymbol{\nabla} \times(\mathbf{J} \times \mathbf{B})$ go to zero at infinity (here this will mean a boundary farther than the magnetopause). If a magnetic configuration is in equilibrium with isotropic pressure, then $\boldsymbol{\nabla} \times \mathbf{G}=0$, and therefore $\boldsymbol{\nabla} \times \mathbf{G}$ represents in a general case the departure from equilibrium with isotropic $P$.

\subsection{1-D pressure profile from an empirical field model}

As mentioned, Horton et al. [1993] computed 2-D profiles of isotropic $P$ for T87 and T89 by performing simple quadratures of $\partial P / \partial u$ and $\partial P / \partial v$ on the equatorial plane, where $u$ and $v$ represent two coordinates defining the plane. This method is however mathematically unsound, because $P$ at a particular point depends on the path of integration, as we will show below.

In applying Eq. 10 on the equatorial plane of a north-south symmetric empirical model, the absence of exact force balance will dictate $\boldsymbol{\nabla} \times \mathbf{G} \neq 0$ and therefore no function $P$ can be found on the plane that satisfies $\mathbf{J} \times \mathbf{B}=\nabla P$. The implication of this can be seen if we assume $\mathbf{J} \times \mathbf{B}=\boldsymbol{\nabla} P$, and consider its component in the directions of $(\mathbf{B} \times \nabla \psi)$ and $(\mathbf{B} \times$ $\nabla \alpha$ ), respectively:

$$
\begin{gathered}
\mathbf{J} \cdot \boldsymbol{\nabla} \psi=\boldsymbol{\nabla} \cdot\left[(\boldsymbol{\nabla} \psi)^{2} \boldsymbol{\nabla} \alpha-(\boldsymbol{\nabla} \alpha \cdot \boldsymbol{\nabla} \psi) \boldsymbol{\nabla} \psi\right]=-\frac{\partial P}{\partial \alpha}, \\
(11) \\
\mathbf{J} \cdot \boldsymbol{\nabla} \alpha=\boldsymbol{\nabla} \cdot\left[(\boldsymbol{\nabla} \alpha \cdot \boldsymbol{\nabla} \psi) \boldsymbol{\nabla} \alpha-(\boldsymbol{\nabla} \alpha)^{2} \boldsymbol{\nabla} \psi\right]=\frac{\partial P}{\partial \psi},
\end{gathered}
$$

as obtained by [e.g., Birn et al., 1977; Cheng, 1995]. The problem of finding $P$ from Eqs. 11 and 12 is not well posed for fields that do not satisfy force balance, since the function $P$ defined by Eqs. 11 and 12 is not analytic (regular)! More exactly,

$\left.\left.\frac{\partial}{\partial \psi}\left(\frac{\partial P}{\partial \alpha}\right)=-\frac{\partial}{\partial \psi}(\mathbf{J} \cdot \nabla \psi)\right) \neq \frac{\partial}{\partial \alpha}(\mathbf{J} \cdot \nabla \alpha)\right)=\frac{\partial}{\partial \alpha}\left(\frac{\partial P}{\partial \psi}\right)$

Therefore, the equivalent of Eqs. 11 and 12 in any other coordinate system cannot be used to obtain 2-D $P$ profiles from magnetic configurations without force balance. Trying to integrate Eqs. 11 and 12 starting from a point where $P$ is chosen will give values dependent on the integration path! To see whether this is the case for the T96 model, we have used Eqs. 11 and 12 to compute $P$ at point B in Fig. 3.1 starting from the farthest point $\mathrm{A}$, following two paths: (1) the direct path, along constant midnight $\alpha$, and (2), through $A_{1}$ and $A_{2}$. The results obtained for $P_{B}$, starting from $P_{A}=0.036 \mathrm{nPa}$, are $4.24 \mathrm{nPa}$ and $4.57 \mathrm{nPa}$ for integration done along paths (1) and (2), respectively. This difference shows that there is no regular (analytic) $P$ with $\nabla P=\mathbf{J} \times \mathbf{B}$, therefore using Eqs. 11 and 12 to obtain 2-D $P$ profiles from empirical model fields is not a physically well-posed problem.

In an east-west symmetric model however, on the Sun-Earth line the problem is well-posed due to $\partial P / \partial \alpha$ $=0$ there. Therefore, $\mathbf{J} \times \mathbf{B}$ can be integrated on that line due to the symmetry of the problem, however the resulting $P$ cannot be extended to other local times.

There is another very important use for equations 11 and 12 however. They can be construed as coupled 2-D elliptic equations for $\alpha$ and $\psi$ on constant $\psi$ and $\alpha$ surfaces, respectively. By solving them numerically for example, a self-consistent magnetospheric picture could be obtained in 3-D if the $P$ profile is prescribed on one plane. This approach, with $P=P(\psi)$, has been used by Cheng [1995] to compute a 3-D magnetospheric equilibrium. 


\subsection{Rigorous way of obtaining $P$ : Poisson equation}

With the 2-D direct integration of $\nabla P=\mathbf{J} \times \mathbf{B}$ seen to be an ill-posed problem, we concentrate on the rigorous solution for $P$ (and $\mathbf{G})$ ) defined by the Helmholtz decomposition of $\mathbf{J} \times \mathbf{B}$, Eq. 10 .

The quantities $P$ and $\mathbf{G}$ can be formally expressed as [e.g., Arfken, 1985]

$$
\begin{aligned}
P(\mathbf{r}) & =\int_{V} \frac{\boldsymbol{\nabla} \cdot(\mathbf{J} \times \mathbf{B})}{4 \pi\left|\mathbf{r}-\mathbf{r}^{\prime}\right|} d^{3} r^{\prime}, \\
\mathbf{G}(\mathbf{r}) & =\int_{V} \frac{\boldsymbol{\nabla} \times(\mathbf{J} \times \mathbf{B})}{4 \pi\left|\mathbf{r}-\mathbf{r}^{\prime}\right|} d^{3} r^{\prime},
\end{aligned}
$$

where the volume integrals have to be performed over the whole spatial domain with non-zero $\boldsymbol{\nabla} \cdot(\mathbf{J} \times \mathbf{B})$ and $\boldsymbol{\nabla} \times(\mathbf{J} \times \mathbf{B})$, respectively. Eq. 14 shows that $\boldsymbol{\nabla} \times(\mathbf{J} \times \mathbf{B})$ acts as a "source" for $\mathbf{G}$, and that $\mathbf{G}$ could still be considerable at point $\mathbf{r}$ even if the $\boldsymbol{\nabla} \times(\mathbf{J} \times \mathbf{B})$ is small locally. One also sees that in order to find $\boldsymbol{\nabla} \times \mathbf{G}$ one needs to know $\boldsymbol{\nabla} \times(\mathbf{J} \times \mathbf{B})$ everywhere.

One method for obtaining $P$ is by directly computing it from Eq. 13. That however involves an integration over the whole region where the integrand is non-zero (i.e. the whole magnetosphere), and is not practical, as we would need to know $\boldsymbol{\nabla} \cdot(\mathbf{J} \times \mathbf{B})$ everywhere! A more tractable way of finding $P$ is observing that

$$
\nabla^{2} P=\nabla \cdot(\mathbf{J} \times \mathbf{B})
$$

which is an elliptic Poisson-type equation, requiring appropriate boundary conditions for uniqueness of the solution. After solving Eq. 15 one can obtain $\boldsymbol{\nabla} \times \mathbf{G}$ from Eq. 10, and then a comparison between both $\boldsymbol{\nabla} P$ and $\boldsymbol{\nabla} \times \mathbf{G}$ with $\mathbf{J} \times \mathbf{B}$ will indicate how far from an equilibrium with isotropic $P$ the empirical field is. Eq. 15, was mentioned by Horton et al. [1993] in their study of the T87 and T89 models, but the authors did not present $P$ solutions.

In this paper we will investigate Eq. 15 on the equatorial plane, but while only considering isotropic $P=P(\psi, \alpha)$, we will take into account components due to the dependence on the third coordinate (along the field) in the operators $\boldsymbol{\nabla}$ and $\nabla^{2}$. In our $(\psi, \alpha, \chi)$ system, the LHS of Eq. 15 is:

$$
\begin{aligned}
\nabla^{2} P= & \boldsymbol{\nabla} \cdot(\boldsymbol{\nabla} P)=\frac{1}{\mathcal{J}}\left\{\frac{\partial}{\partial \psi}\left[\mathcal{J}(\boldsymbol{\nabla} P)^{\psi}\right]+\right. \\
& \left.+\frac{\partial}{\partial \alpha}\left[\mathcal{J}(\boldsymbol{\nabla} P)^{\alpha}\right]+\frac{\partial}{\partial \chi}\left[\mathcal{J}(\boldsymbol{\nabla} P)^{\chi}\right]\right\},
\end{aligned}
$$

where the superscripts denote the contravariant components of $\nabla P$. Because $P=P(\psi, \alpha), \partial P / \partial \chi=0$ and one might be tempted to drop the last term in Eq. 16. However, that third term contains the contravariant component of $\boldsymbol{\nabla} P,:(\boldsymbol{\nabla} P)^{\chi}=\boldsymbol{\nabla} P$. $\boldsymbol{\nabla} \chi=(\partial P / \partial \psi)(\boldsymbol{\nabla} \psi \cdot \boldsymbol{\nabla} \chi)+\partial P / \partial \alpha(\boldsymbol{\nabla} \alpha \cdot \boldsymbol{\nabla} \chi)$, whose derivative with respect to $\chi$ is non-zero, because $\partial / \partial \chi(\boldsymbol{\nabla} \psi \cdot \boldsymbol{\nabla} \chi) \neq 0$ and $\partial / \partial \chi \boldsymbol{\nabla} \alpha \cdot \boldsymbol{\nabla} \chi \neq 0$. The third term in Eq. 16 - the equivalent of $\partial^{2} P / \partial Z^{2}$ in a Cartesian system - is thus not zero, with its values actually found to be quite significant on the equatorial plane. Again we note a benefit of using the flux coordinate system, as the term is explicitly written in terms of $\partial P / \partial \psi$ and $\partial P / \partial \alpha$ in this system, however in a Cartesian system further calculation would be needed to express $\partial^{2} P / \partial Z^{2}$ in terms of $\partial P / \partial X$ and $\partial P / \partial Y$, based on the constancy of $P$ along the field line.

With regard to the boundary conditions for the Poisson equation, it is evident that they cannot be obtained from the empirical model itself, and additional assumptions are needed. One choice is Dirichlet conditions on the inner and outer flux surfaces, with values for $P$ chosen on those surfaces as given by the so-called Spence-Kivelson empirical formula [Spence and Kivelson, 1993]. In the $\alpha$ coordinate, the boundary condition is periodic: $P(\alpha+2 \pi)=P(\alpha)$.

\subsection{Departure from equilibrium: $\nabla \times \mathrm{G}$}

The main goal of our work is to investigate the T96 on physical grounds, searching for how close the model can be in force balance with an isotropic pressure distribution.

Several such tests have been performed [Walker and Southwood, 1982; Horton et al., 1993; Cao and Lee, 1994] for earlier models such as T87 and T89 . These studies mainly applied a "momentum balance test", investigating $\boldsymbol{\nabla} \times(\mathbf{J} \times \mathbf{B})$, which should vanish if an exact quasi-static equilibrium with $\nabla P=\mathbf{J} \times \mathbf{B}$ describes the system. The above parameter was examined in itself by Walker and Southwood [1982], and its magnitude compared with $\boldsymbol{\nabla} \cdot(\mathbf{J} \times \mathbf{B})$ or $|\mathbf{J} \times \mathbf{B}|$ by Horton et al. [1993], Cao and Lee [1994], respectively.

A main conclusion reached by Cao and Lee [1994] by examining the parameter $\epsilon=\mathrm{R}_{\mathrm{E}}|\boldsymbol{\nabla} \times(\mathbf{J} \times \mathbf{B})| / \mid \mathbf{J} \times$ $\mathbf{B} \mid$ was that T87 was "almost isotropic". In the case of an equilibrium with isotropic $P$ however, one expects the field-aligned current as obtained from Vasyliunas relation (Eq. 7) to coincide with the actual current directly obtained from Ampere's law $\boldsymbol{\nabla} \times \mathbf{B}=\mathbf{J}$. Such 
a test was performed by Ding et al. [1994] in the T87 case, however they obtained widely different results. By using Vasyliunas relation (in which they replaced $\nabla P$ by $\mathbf{J} \times \mathbf{B}$ ), they found much larger (by more than one order of magnitude) currents than the actual ones in T87 (which are very small as mentioned). This result is suspicious, and the question to be answered is: what gives rise to "field-aligned currents" using Vasyliunas relation where $J_{\|}=0$ ? The most likely possibility that might explain the contradiction in the results of Ding et al. [1994] is that the T87 model is not really "almost isotropic", but that somehow the magnitude of $\boldsymbol{\nabla} \times(\mathbf{J} \times \mathbf{B})$ is not a good test for locally characterizing the isotropy.

Indeed, each of the past approaches suffers from one or more of the following problems: (1) the quantity $\boldsymbol{\nabla} \cdot(\mathbf{J} \times \mathbf{B})$ can be very small, resulting in large values of the ratio $|\boldsymbol{\nabla} \times(\mathbf{J} \times \mathbf{B})| /|\boldsymbol{\nabla} \cdot(\mathbf{J} \times \mathbf{B})|$ examined by Horton et al. [1993]; (2) the parameter $\epsilon$ computed by Cao and Lee [1994] for T87 and T89 cannot be applied to T96, as it will experience large variations at the thin field-aligned current sheet boundaries, and (3) most importantly, $\boldsymbol{\nabla} \times(\mathbf{J} \times \mathbf{B})$ is not the best indicator of departure from an equilibrium with isotropic $P$. Indeed, from Eq. 10 one finds $\boldsymbol{\nabla} \times(\mathbf{J} \times \mathbf{B})=\boldsymbol{\nabla} \times(\boldsymbol{\nabla} \times \mathbf{G})$. However, in order to quantify the departure from equilibrium with an isotropic pressure, it is not sufficient to know $\boldsymbol{\nabla} \times(\boldsymbol{\nabla} \times \mathbf{G})$, because it is necessary to compare $\boldsymbol{\nabla} P$ and $\boldsymbol{\nabla} \times \mathbf{G}$. Because $\boldsymbol{\nabla} \times \mathbf{G}=\mathbf{J} \times \mathbf{B}-\boldsymbol{\nabla} P$ is the true measure of the departure from equilibrium, the degree of this departure in an empirical model cannot be characterized just by inspection of $\boldsymbol{\nabla} \times(\mathbf{J} \times \mathbf{B})$.

\section{Numerical Method and Computational Domain}

From a computational point of view it is desirable that one be able to describe different regions of the magnetosphere with different spatial resolutions. For this purpose, we introduce two computational functions $\rho_{c}, \zeta_{c}$, such that $\psi=\psi\left(\rho_{c}\right)$, $\alpha=\alpha\left(\zeta_{c}\right)$, and therefore the $\mathbf{B}$ is expressed as $\mathbf{B}=\left(d \psi / d \rho_{c}\right)\left(d \alpha / d \zeta_{c}\right)\left(\nabla \rho_{c} \times \nabla \zeta_{c}\right)$. Accuracy can be increased where that is needed (e.g. in regions of steep gradients), by a judicious choice of $\rho_{c}$ and $\zeta_{c}$. With $\chi_{c}=\chi$, we have a "computational flux coordinate system," with the computational coordinates defined in the intervals $0 \leq \rho_{c} \leq 1,0 \leq \zeta_{c} \leq 2 \pi$, and $0 \leq \chi_{c} \leq \pi$ [Cheng, 1995].

One subtle issue needs to be mentioned here. Un- like in T87 or T89, the field in T96 is not divergencefree by construction. In particular, the field due to the Birkeland currents is computed by separate best fit analytical approximations for each component $B_{x}$, $B_{y}$ and $B_{z}$, thus precluding an exact $\boldsymbol{\nabla} \cdot \mathbf{B}=0[$ Tsyganenko and Stern, 1996]. A significantly non-zero $\boldsymbol{\nabla} \cdot \mathbf{B}$ would be physically problematic and no Euler potential representation would be accurate, as the field defined by $\mathbf{B}=\boldsymbol{\nabla} \psi \times \nabla \alpha$ intrinsically satisfies $\boldsymbol{\nabla} \cdot \mathbf{B}=0$. We have however computed $\boldsymbol{\nabla} \cdot \mathbf{B}$ on and in the vicinity of the equatorial plane using a very fine Cartesian grid and obtained values $\mathrm{R}_{\mathrm{E}} \boldsymbol{\nabla} \cdot \mathbf{B} / B$ on the order of $10^{-2}$ or less, thus verifying the claim by Tsyganenko and Stern [1996] that an approximate $\boldsymbol{\nabla} \cdot \mathbf{B} \approx 0$ should still hold with sufficient accuracy. Therefore, $\mathbf{B}=\boldsymbol{\nabla} \psi \times \nabla \alpha$ is an accurate representation of the T96 field.

Since the field at the Earth's surface can be assumed to be overwhelmingly due to internal Earth sources [Tsyganenko, 1990] and only taking into account the dipole expansion, from the dipole axisymmetry we have $\alpha \equiv \phi$ on the Earth's surface as seen before. The value of $\psi$ on the Earth's surface on the other hand is simply related to the poloidal flux of a dipole field [e.g., Cheng, 1995]: $\psi=-B_{D} \mathrm{R}_{\mathrm{E}}^{2} \sin ^{2} \theta$, where $B_{D}$ and $\mathrm{R}_{\mathrm{E}}$ are the equatorial dipole field on the Earth's surface and the Earth radius, respectively, while $\theta$ represents the colatitude.

For simplicity, we will only consider the case of zero tilt of the geodipole axis. For a certain set of parameters in T96 we trace various field lines starting from the Earth's surface, taking advantage of the known values for $\psi$ and $\alpha$ there. The footpoints of the traced field lines cover the Earth surface in a chosen latitude interval, with footpoint density that can be varied by a judicious choice of $\rho_{c}(\psi)$. For each latitude (i.e., each $\psi$ ) we trace field lines with footpoints on Earth in the whole $360^{\circ}$ longitude range. Very good resolution in the step size is needed for the tracing [Peymirat and Fontaine, 1999], because a small difference in the latitudes of the footpoints of two different field lines on the night side can translate into a difference of a few $\mathrm{R}_{\mathrm{E}}$ on the equatorial plane. To this end, our tracing was performed using an adaptive Runge-Kutta technique, with an average step size of $200 \mathrm{~km}$.

By applying the described tracing method we obtained a grid of $201 \times 201(\psi, \alpha)$ values. On each traced line, we retained 21 equally-spaced points within $2 \mathrm{R}_{\mathrm{E}}$ from the equatorial plane, to have an accurate calculation of partial derivatives $\partial / \partial \chi$. The 
obtained grid is thus characterized by an average spatial separation of $0.15 \mathrm{R}_{\mathrm{E}}$ in the radial direction, $0.5 \mathrm{R}_{\mathrm{E}}$ in the azimuthal direction and $0.1 \mathrm{R}_{\mathrm{E}}$ along the field line. The largest latitude (thus largest $\psi$ ) was chosen such that the domain did not extend past $35 R_{\mathrm{E}}$ in the night side, because very few points are available there in the database used in T96 [Tsyganenko, 1995]. At the other end, we have chosen $3.5 \mathrm{R}_{\mathrm{E}}$ to be the inner domain boundary at midnight, as certain boundary conditions for $P$ later discussed are not valid closer than that.

Finally we need to underscore yet another advantage of the Euler potential approach to representing the T96 field: the fitting of the model field to the data was done [Tsyganenko, 1995] by considering least squares for the differences of unit direction vectors $\mathbf{b}=\mathbf{B} / B$, rather than the full vectors $\mathbf{B}$, thus making T96 a better choice for mapping compared to previous models. Therefore, a depiction of T96 by using field-tracing techniques seems only natural.

\section{Results for Pressure From Quiet-time T96}

\subsection{Pressure obtained by direct $1-D$ integra- tion on the Sun-Earth line}

As mentioned, the problem of integrating $\nabla P=$ $\mathbf{J} \times \mathbf{B}$ with an empirical model field only makes sense on the Sun-Earth line, as at other azimuths $P$ is not a regular (analytic) function. In this subsection we present results for $P$ (denoted by $P_{1}$ from here on) obtained by integrating Eq. 12 for $\partial P / \partial \psi$ on the Sun-Earth axis. The present study, the first to our knowledge for T96, is similar to previous work investigating 1-D force balance with isotropic pressure in earlier empirical models [e.g., Spence et al., 1987; Kan et al., 1992]. We have done the calculation for a quiet-time state, with T96 described by $D S T=0$, $P_{d y n}=0.55 \mathrm{nPa}, B_{y I M F}=0$ and $B_{z I M F}=10 \mathrm{nT}$, typical for a quiet time. With these parameters, the outer limit at $36 \mathrm{R}_{\mathrm{E}}$ corresponds to a latitude of $73.9^{\circ}$ at midnight, while the inner boundary at $3.5 \mathrm{R}_{\mathrm{E}}$ corresponds to a midnight latitude of $57.7^{\circ}$.

Eq. 12 only determines $P_{1}$ up to a constant, which was chosen by letting the value of $P_{1}$ at $36 R_{E}$ at midnight to be the value from the Spence-Kivelson empirical formula [Spence and Kivelson, 1993] . The farthest point was chosen as reference point because $P_{1}$ is smallest there and so even if there was an error in the value chosen there, it will translate into a much smaller relative error for $P_{1}$ closer to Earth.

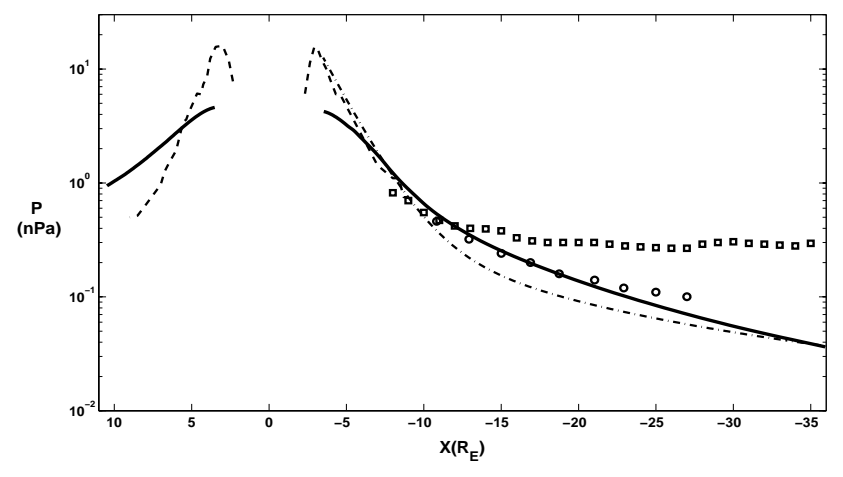

Figure 4. Profile of $P_{1}$, obtained by integrating $\boldsymbol{\nabla} P_{1}=\mathbf{J} \times \mathbf{B}$ from T96 on the Sun-Earth line (solid lines); also shown: average pressure from AMPTE/CCE up to $9 \mathrm{R}_{\mathrm{E}}$ [Lui and Hamilton, 1992] (dashed lines); DMSP data mapped by Wing and Newell [1998] (squares); GEOTAIL plasma sheet pressure [Hori et al., 2000] (circles); and finally, the Spence-Kivelson empirical profile [Spence and Kivelson, 1993] (dash-dotted line).

In Fig. 4, we plot the obtained $P_{1}$ profiles on the Sun-Earth line together with several observations, as well as the Spence-Kivelson formula, i.e. $P(\mathrm{nPa})=89 e^{-0.59|X|}+8.9|X|^{-1.53}$. Most observations only provide the proton pressure, however this should not present a problem, as the contribution of other ions is negligible in quiet times [e.g., Lui and Hamilton, 1992], while the electron pressure is also much smaller (about $1 / 7$ th of the proton pressure [Baumjohann et al., 1989]).

For the innermost part of the nightside magnetosphere, the comparison is made with data obtained by AMPTE/CCE [Lui and Hamilton, 1992]. Since Lui and Hamilton [1992] compute both $P_{\perp}$ and $P_{\|}$from the particle distribution function, for the purpose of this comparison we plot their average pressure, defined as $\langle P\rangle=\left(2 P_{\perp}+P_{\|}\right) / 3$. It can be seen that from $3.5 \mathrm{R}_{\mathrm{E}}$ to about geosynchronous orbit $\left(6.6 \mathrm{R}_{\mathrm{E}}\right)$, our computed $P_{1}$ tends to be lower than the average observed value. This fact might be explained by the argument of Lui and Hamilton [1992] that if significant anisotropy exists but the pressure is nevertheless computed from force equilibria with the isotropic assumption, the obtained values can underestimate the real pressure by as much as a factor of 3 .

For comparison at larger distances, we plot GEOTAIL plasma sheet pressure data [Hori et al., 2000], as well as ionospheric data from DMSP mapped, us- 
ing T89, by Wing and Newell [1998]. The GEOTAIL data is averaged in the domain $|Y|<2 \mathrm{R}_{\mathrm{E}}$, while the mapped profile from DMSP is averaged over $-10 \mathrm{R}_{\mathrm{E}}<Y<10 \mathrm{R}_{\mathrm{E}}$. We observe that our computed $P_{1}$ is quite consistent with the GEOTAIL observations, which suggests, within the assumption of equilibrium, that T96 could be in 1-D force balance along the midnight meridian in the equatorial plane. The computed $P_{1}$ is significantly lower than the values of Wing and Newell [1998], which are basically constant from 20 to $40 \mathrm{R}_{\mathrm{E}}$. It has been discussed however [Wang and Lyons, 2001] that the profile of Wing and Newell [1998] is larger than other observations as well, and we believe that this might be due to the fact that Wing and Newell [1998] use T89 to map $P$ from the ionosphere, since T89 has been found to be excessively stretched in the tail [Peredo et al., 1993; Rostoker and Skone, 1993], and less reliable than T87 in predicting accurate $\mathbf{B}$ in the region from 10 to $20 \mathrm{R}_{\mathrm{E}}$. If this is the case, the $P$ shown in [Wing and Newell, 1998] beyond $15 \mathrm{R}_{\mathrm{E}}$ is in fact "translated" from closer distances. The findings of Lui et al. [1994] support this interpretation because their 1-D integration of the magnetic force in the T89R model (a modified T89 different from the original only very close to Earth) produced a $P$ profile almost constant between 15 and $35 \mathrm{R}_{\mathrm{E}}$. However this result is not obtained when another model (derived from T87), is considered.

We now look at the dayside region, which has been much less analyzed before. Besides our calculation, we plot the average profile from AMPTE/CCE $[L u i$ and Hamilton, 1992], again defining $\langle P\rangle=\left(2 P_{\perp}+\right.$ $\left.P_{\|}\right) / 3$. As opposed to the situation on the night side, there is less consistency between the data and our obtained $P_{1}$. The observed pressure on the day-side peaks at a higher value, and has a sharper gradient. The inconsistency could be explained by anisotropy, which is found to be rather large throughout the dayside region [Lui and Hamilton, 1992].

\subsection{Solution for $P$ from the Poisson equation; $\nabla P$ vs. $\nabla \times \mathbf{G}$}

In this section we present the 2-D solution for $P$ from Eq. 15, which we denote by $P_{2}$ from here on. The purpose of the study is to obtain $\boldsymbol{\nabla} P_{2}$ and $\boldsymbol{\nabla} \times \mathbf{G}$, and to compare them, which would provide an indication of how well the T96 model supports a "quasi-static equilibrium" with an isotropic pressure. We have solved Eq. 15 numerically for the same quiet-time state as before using Dirichlet boundary conditions, and Fig. 5 shows equatorial contours of the obtained

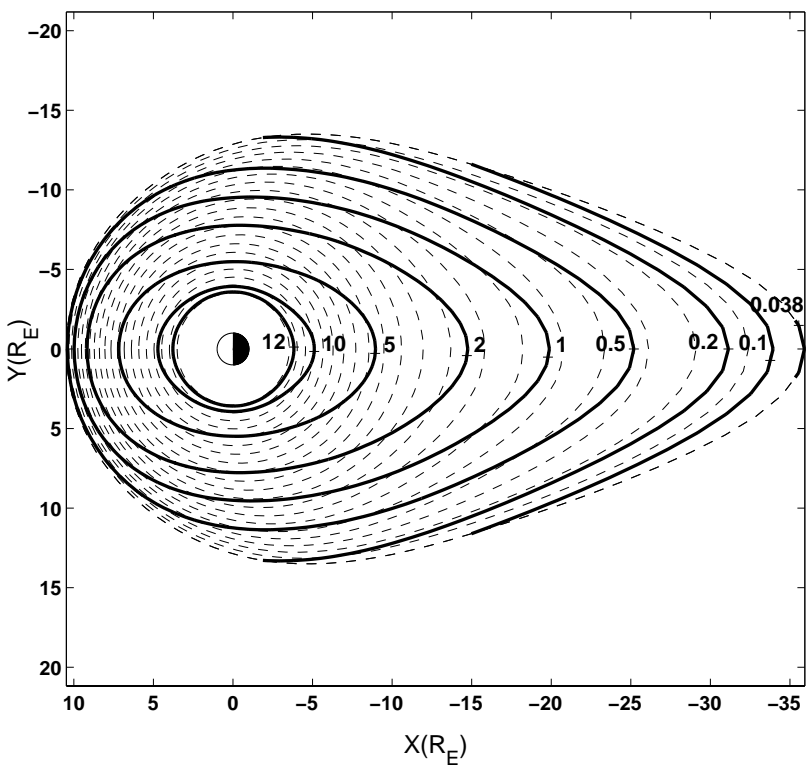

Figure 5. Contours of equal $P_{2}(\mathrm{nPa})$ on the equatorial plane from Eq. 15 with $P_{b}=P_{b}(r)$ boundary conditions (solid lines); constant $\psi$ contours are also shown (dashed lines)

$P_{2}$. We also present in subplot (a) of Fig. 6 the profile of $P_{2}$ along the Sun-Earth line, along with the previous profile $P_{1}$ obtained by integrating Eq. 12 along that line, as well as the empirical Spence-Kivelson profile.

Next, with $P_{2}$ known, we computed $\nabla P_{2}$ and $\nabla \times$ $\mathbf{G}=\mathbf{J} \times \mathbf{B}-\boldsymbol{\nabla} P_{2}$ as defined in Eq. 10. Obviously, before performing the calculation, one might suspect that due to the large discrepancy seen in subplot (a) of Fig. 6 between the computed $P_{2}$ and the SpenceKivelson formula (which as seen in Fig. 4 is quite similar to the $P_{1}$ profile), both $\boldsymbol{\nabla} P_{2}$ and $\boldsymbol{\nabla} \times \mathbf{G}$ will be found to be quite large and different from $\mathbf{J} \times \mathbf{B}$. This is indeed the case, as seen in subplots (b) and (c) of Fig. 6, where the values of $\boldsymbol{\nabla} P_{2}, \boldsymbol{\nabla} \times \mathbf{G}$ and $\mathbf{J} \times \mathbf{B}$ are shown along the Sun-Earth line. It is seen that everywhere $|\mathbf{J} \times \mathbf{B}|$ is smaller than both $\left|\nabla P_{2}\right|$ and $|\boldsymbol{\nabla} \times \mathbf{G}|$. The large values of $|\boldsymbol{\nabla} \times \mathbf{G}|$ suggest that the T96 model field does not support an equilibrium with isotropic $P$ constant along the field line, not even in the plasma sheet region.

\subsubsection{Effect of boundary conditions}

It would appear that the particular dependence of $P_{2}$ obtained from Eq. 15 on $\psi$ and $\alpha$ might be due to our choice of boundary conditions, i.e. $P_{b}=P_{b}(r)$. 

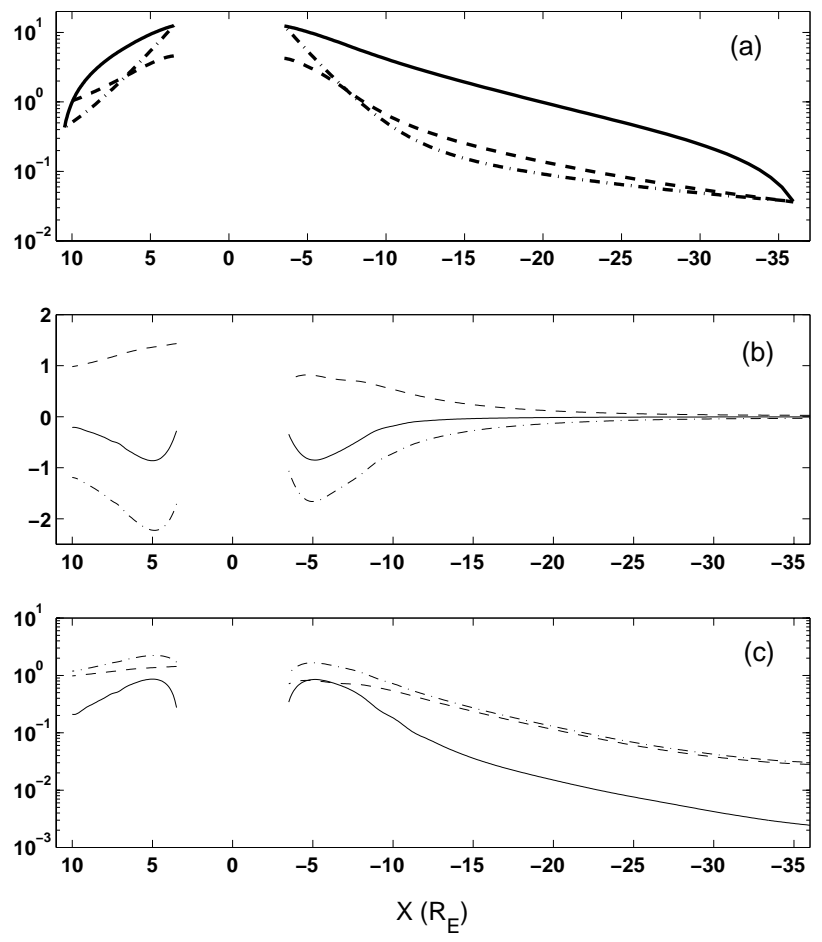

Figure 6. (a) $P_{2}(\mathrm{nPa})$ obtained with $P_{b}=P_{b}(r)$ boundary conditions, shown only on the Sun-Earth axis (solid line); also, $P_{1}$ obtained by $1-\mathrm{D}$ integration (dashed line) and the Spence-Kivelson profile (dashdotted line); (b) Values on the Sun-Earth axis of magnetic force $\mathbf{J} \times \mathbf{B}$ (solid lines), $\boldsymbol{\nabla} P_{2}$ (dash-dotted lines) and $\boldsymbol{\nabla} \times \mathbf{G}$ (dashed lines); all three quantities are in the radial direction, and are given in $n P a / R_{E} ;(c)$ same quantities as in (b), but logarithmic plot of their absolute values.

However, there are several indications that point to the contrary, namely that the shape of the $P$ distribution is due to the source term $\boldsymbol{\nabla} \cdot(\mathbf{J} \times \mathbf{B})$. First, it is evident from Fig. 5 that the $P=P(r)$ behavior is valid only very close to the inner and outer $\psi$ boundaries, and that the 2-D $P_{2}$ distribution drastically changes away from boundaries. Furthermore, we present in Fig. 7 the result for $P_{2}$ obtained from Eq. 15 with a different boundary condition, $P_{b}=P_{b}(\psi)$. The result is seen to be very similar to the previously obtained $P_{2}$, except within $1 \mathrm{R}_{\mathrm{E}}$ from the outer $\psi$ boundary. We also present in Fig. 8 the profile on the Sun-Earth axis of $P_{2}$ obtained from Eq. 15 with a lower value of $P_{2}$ on the inner boundary at midnight, $P_{2}=4.24 \mathrm{nPa}$, the same as $P_{1}$ there. For this case, at different $\alpha$ values, $P_{2}$ on the boundary was obtained

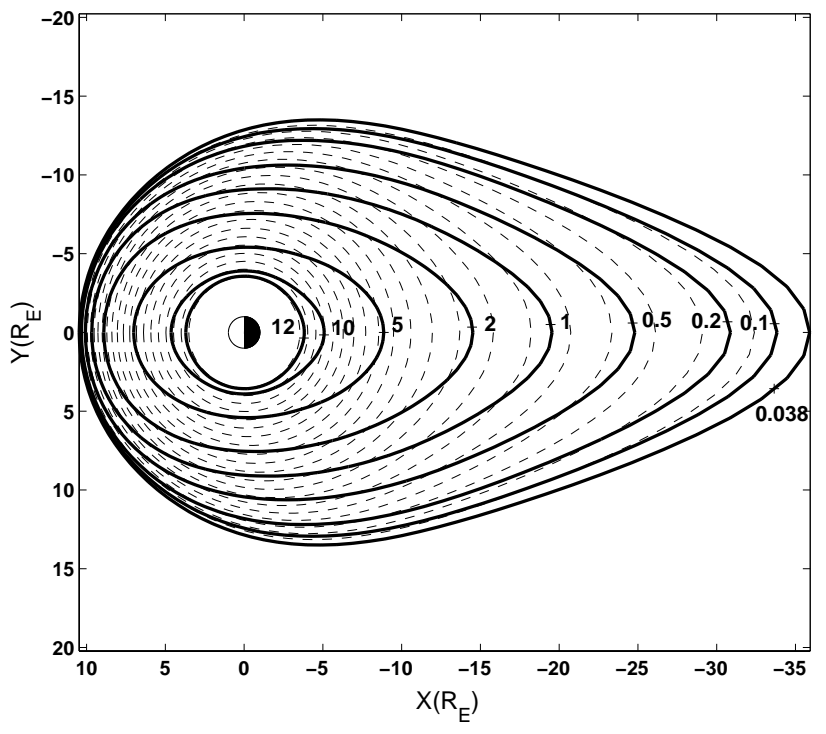

Figure 7. Contours of equal $P_{2}(\mathrm{nPa})$ on the equatorial plane from Eq 15 with $P_{b}=P_{b}(\psi)$ boundary conditions (solid lines); constant $\psi$ contours are also shown (dashed lines)

by integrating $\partial P / \partial \alpha$ from Eq. 11 on both the inner and outer $\psi$ surfaces.

We limit our presentation to Dirichlet boundary conditions. There is a physical rationale however for trying to impose Neumann conditions, as one might think that by choosing $\nabla P_{2} \approx \mathbf{J} \times \mathbf{B}$ on one or both boundaries (i.e. $\boldsymbol{\nabla} \times \mathbf{G} \approx 0$ there), one could find a state in which $\boldsymbol{\nabla} \times \mathbf{G}$ would be minimal throughout the domain. However, the fact that no $P$ exists such that $\nabla P=\mathbf{J} \times \mathbf{B}$ also makes implementation of this approach impossible — the various $\partial P / \partial \psi$ values are not differentiable in $\alpha$, and thus the problem is not well-posed physically. Besides, even if one tried to "smooth" $\partial P / \partial \alpha$, the problem with Neumann conditions on both boundaries would still not be well posed. This is due to the well-known fact [e.g., Hackbusch, 1992] that the problem with Neumann conditions on both boundaries is well-posed only if $\int_{V} \boldsymbol{\nabla} \cdot(\mathbf{J} \times \mathbf{B}) d^{3} r=\int_{\Gamma} \partial P_{2} / \partial \mathbf{n} \cdot d \mathbf{S}$, where the integral over $V$ represents the integral over the whole domain, while $d \mathbf{S}=\mathbf{n} d S$ is the directed surface element on the boundaries.

\subsection{Discussion}

A significant dissimilarity is seen in the radial dependence along the Sun-Earth axis of $P_{1}$ obtained by direct integration of Eq. 12 vs. $P_{2}$ from the Poisson 


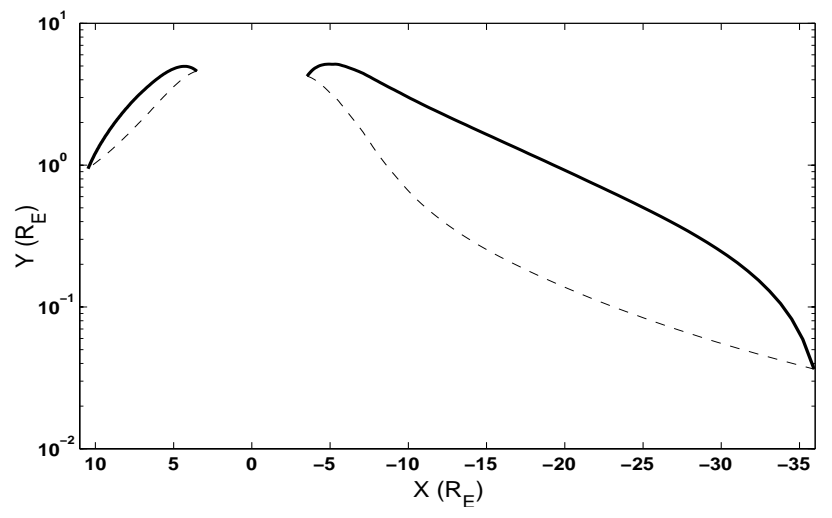

Figure 8. Sun-Earth axis profile of $P_{2} \quad(\mathrm{nPa})$ obtained from Eq. 15 with boundary conditions $P_{\text {inner }}=4.24 \mathrm{nPa}$ taken equal to $P_{1}$ at noon and midnight, with $\alpha$ behavior from integrating $\partial P / \partial \alpha$ in Eq. 11 along the inner and outer $\psi$ surfaces (solid lines); $P_{1}$ profile from 1-D integration also shown (dashed lines);

equation, Eq. 15, as is depicted in Figure 4, subplot (a) of Fig. 6 and Fig. 8. It is evident that $P_{2}$ is significantly larger than $P_{1}$ at all radial distances, with the exception of the farthest end point, where the value from the Spence-Kivelson formula is imposed. Both profiles however show a monotonic decrease with $\psi$ : $\partial P / \partial \psi<0$ (and so $\partial P / \partial r<0$, consistent with observations tail-ward of $3.5 \mathrm{R}_{\mathrm{E}}$ [e.g., Lui et al., 1994]). Nevertheless, $P_{2}$ exhibits lower $\partial^{2} P / \partial r^{2}>0$, i.e. the change in $\nabla P_{2}$ is much lower than both $\nabla P_{1}$ and the observed $\nabla P$. Thus $P_{2}$ obtained from Eq. 15 does not agree with findings [Spence et al., 1989; Lui et al., 1994] of a large change in $\nabla P$ in the "transition region" between the dipole-like and tail-like field configurations - indeed, the largest $\nabla P_{2}$ consists in the significant drop near the outer boundary at $36 \mathrm{R}_{\mathrm{E}}$.

The most relevant comparison between $P_{2}$ and $P_{1}$ is in Fig. 8, where we show $P_{2}$ obtained from Eq. 15 with Dirichlet boundary values taken equal to $P_{1}$ at noon and midnight, and by integrating $\partial P / \partial \alpha$ along the inner and outer $\psi$ surfaces at other local times. Mathematically, the difference between the $P_{2}$ and $P_{1}$ solutions is due to contributions from $\alpha$ and $\chi$ terms in the source term $\boldsymbol{\nabla} \cdot(\mathbf{J} \times \mathbf{B})$, as well as the constraint that $P_{2}$ be constant along the field line. The fundamental physical difference between the two methods is that while the Helmholtz decomposition "filters out" the non-zero curl of $\mathbf{J} \times \mathbf{B}$, the 1 -D method does not. The good correlation between the 1-D profile and ob- servations indicate that the T96 model does a relatively good job in being almost force-balanced with observed pressure in 1-D along the midnight meridian, particularly in the plasma sheet. However, the model is far from being self-consistent in 3-D, as our decomposition of $\mathbf{J} \times \mathbf{B}$ into the curl-free $\nabla P_{2}$ and the divergence-free $\boldsymbol{\nabla} \times \mathbf{G}$ results in unphysical values for $\nabla P_{2}$. The large values of $P_{2}$ make it debatable whether $P_{2}$ alone possesses a physical significance in terms of moments of the particle distribution function, and point to the possibility that $\mathbf{J} \times \mathbf{B}$ obtained from the empirical model might include unphysical contributions that cannot be expressed as the divergence of a pressure tensor $\boldsymbol{\nabla} \cdot \mathrm{P}$.

\section{Computation of Birkeland Currents}

With the $P_{1}$ profile as obtained by direct integration along the tail axis consistent with observations, the use of T96 in 1-D on that axis should not present real problems. This is not the case however if one employs the model away from the Sun-Earth line in a 3-D magnetosphere. Such an application, namely the computation of Birkeland currents using Vasyliunas relation, Eq. 7, is presented in this section. While relatively frequently used in the community, Vasyliunas relation is valid under the underlying assumption of a 3-D force balance between $\mathbf{J} \times \mathbf{B}$ and $\nabla P$. We use Vasyliunas relation, Eq. 7 , where $\nabla P$ will be replaced by $\mathbf{J} \times \mathbf{B}$ from the T96 model, in order to ascertain to what degree the non self-consistency in the empirical model affects the accuracy of the result. As basis of comparison we compute $J_{\|}$in T96 directly from Ampere's law as well: $J_{\|}=\mathbf{b} \cdot(\boldsymbol{\nabla} \times \mathbf{B})$.

The computation using Ampere's law should produce the currents built into T96, so it is useful at this point to recall their topology in that empirical model. The built-in region 1 currents have ionospheric footpoints between $68^{\circ}$ at midnight and $72^{\circ}$ at noon, have maxima on the dayside, and flow in the distant tail to the outer layers of the plasma sheet [Tsyganenko, 1993; Tsyganenko and Stern, 1996]. They are open inside the magnetosphere, closing outside of it in the dayside. The region 2 currents exhibit larger noonmidnight asymmetry, being centered at $61^{\circ}$ at midnight and $66^{\circ}$ at noon. They close inside the magnetosphere through the partial ring current, flowing in a large region from about 5 to $10 R_{E}$.

We perform the computation of $J_{\|}$close to the equatorial plane, because we expect T96 to be closest to force balance in the plasma sheet. In Fig. 9 we 

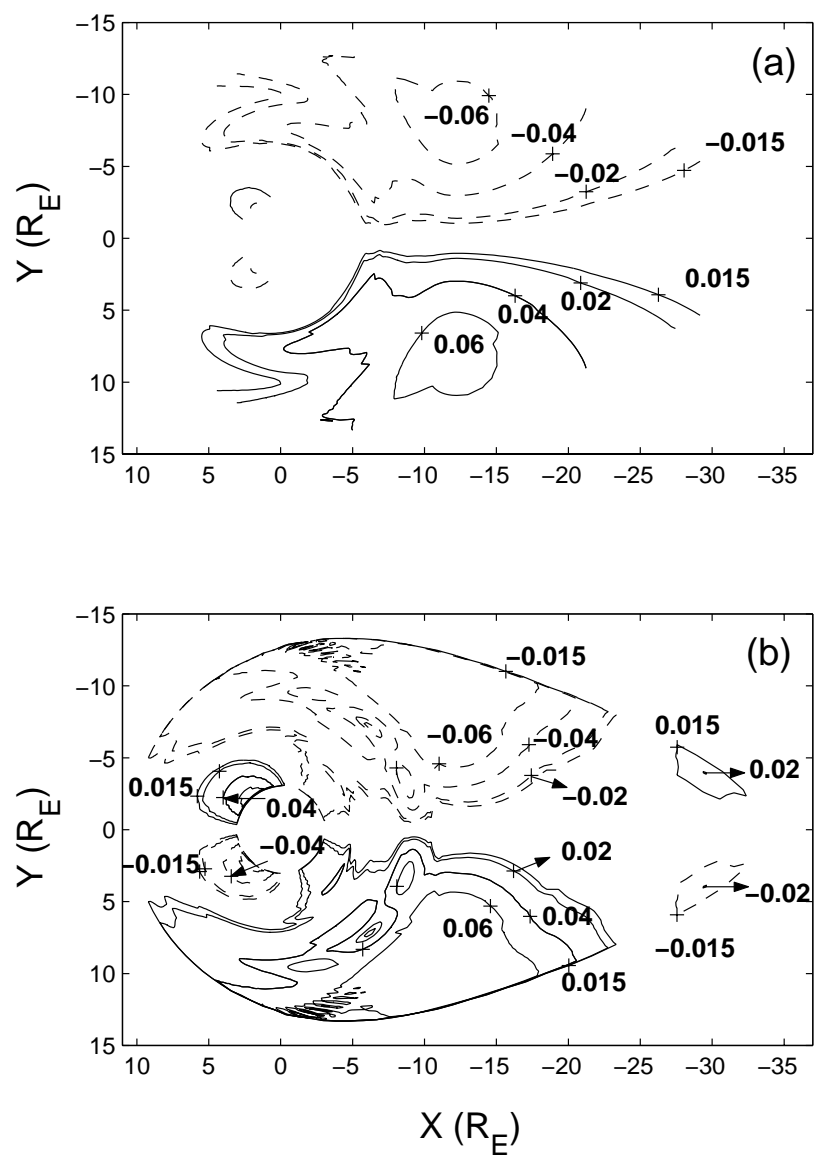

Figure 9. Contours of field-aligned current density (in $\mathrm{nA} / \mathrm{m}^{2}$ ) at $Z=1 \mathrm{R}_{\mathrm{E}}$ above the equatorial plane, obtained directly from T96 by Ampere's law (upper plot) and computed by Vasyliunas relation (lower plot); the solid lines show positive current (out of the equatorial plane), while the dashed lines represent negative current (into the equatorial plane).

present contour plots for the Birkeland currents, at $Z=1 \mathrm{R}_{\mathrm{E}}$ above the equatorial plane. The top subplot of the figure shows $J_{\|}=\mathbf{b} \cdot(\boldsymbol{\nabla} \times \mathbf{B})$. It is purely of region 2 sense and it flows in a relatively large radial domain from $5 \mathrm{R}_{\mathrm{E}}$ to $15 \mathrm{R}_{\mathrm{E}}$. Its largest values are seen to be about $0.06 \mathrm{nA} / \mathrm{m}^{2}$ at around $10 \mathrm{PM}$ and 2 AM local times, respectively. The relatively small values of $J_{\|}$are due to the way the Birkeland currents are built into T96: at small $Z$ in the night side, the region 2 currents flow almost parallel to the equatorial plane as they close via the partial ring current, and therefore their field-aligned component is quite small (see Fig. 6 in [Tsyganenko and Stern, 1996] for the topology of the region 2 Birkeland currents built into T96). In the second subplot of Fig. 9 we show $J_{\|}$computed using Vasyliunas relation. The computed currents in the near-Earth nightside magnetosphere are still of region 2 sense, with a maximum of $0.07 \mathrm{nA} / \mathrm{m}^{2}$, and spatial location roughly the same as the Ampere-obtained currents. However, we also notice the appearance of region 1 sense currents farther in the tail between 25 and $30 \mathrm{R}_{\mathrm{E}}$. This feature is quite problematic because there are no region 1 currents in the picture obtained from Ampere's law - the region 1 currents built into T96 flow on surfaces outside of the plasma sheet, and therefore should not be encountered at $Z=1 \mathrm{R}_{\mathrm{E}}$. We also note the existence of region 1 sense currents on the dayside, very close to Earth. Since at those locations Lui and Hamilton [1992] found significant anisotropies, it can be argued that the force balance there is not complete without non-isotropic terms, and thus Eq. 7 is not valid there. Such an argument is harder to make in the tail at 25 to $30 \mathrm{R}_{\mathrm{E}}$ because observations have shown $P$ to be isotropic.

While not presented here due to space considerations, we have performed the above computations further along the field lines, and obtained larger and larger dissimilarities between $J_{\|}$obtained by the two methods as we approached the ionosphere. Evidently, in the case of a 3 -D force balance with isotropic $P$ one would expect the current profiles obtained by the two methods to be the same. The fact that they are not and especially the appearance of spurious currents reinforces our earlier conclusion that T96 does not support such a force balance.

Recently, Wing and Newell [2000] have applied Vasyliunas relation to model the Birkeland currents in the plasma sheet during quiet times, using the T89 model, together with observed ionospheric $P$ mapped to the plasma sheet using the same T89 [Wing and Newell, 1998]. Since our discussion is pertinent to exactly this type of application, in the following we present a few comments on the work of Wing and Newell [2000]. A first problematic issue in their approach is the mapping, since T89 has no built-in fieldaligned currents, and therefore, as Ding et al. [1994] state, all attempts at detailed mapping using a model which does not include the effects of the Birkeland currents should be viewed with a degree of skepticism. Secondly, the $P$ profiles in conjunction with the $\mathbf{B}$ field used by Wing and Newell [2000] do not represent a self-consistent state. Also, again because T89 has no field-aligned currents, there is almost no geodesic curvature through most of the equatorial plane, as any 
"residual" field-aligned currents are negligible [Ding et al., 1994]; Figure 1 in the paper by Wing and Newell [2000] also shows the magnetic tension mostly oriented Earthward. In these conditions, the contribution to $J_{\|}$comes mainly from the second term in Eq. 8 , which is quite large, as the azimuthal $\nabla P$ in their mapped pressure is itself large. However, such large azimuthal $\nabla P$ cannot be in force balance with the field of T89, as azimuthal changes in magnetic pressure would not be enough to maintain it, and a geodesic curvature is needed, which is only possible in the presence of self-consistent $J_{\|}$. It can be pictured however that if the field of T89 is modified in order to rotate $\boldsymbol{\kappa}$ to balance $\boldsymbol{\nabla} P$, the product $\boldsymbol{\kappa} \times \boldsymbol{\nabla} P$ in Eq. 7 will decrease, thus decreasing the (rather large) values for $J_{\|}$obtained by Wing and Newell [2000] at $Z=0.1 \mathrm{R}_{\mathrm{E}}$.

Another result obtained by Wing and Newell [2000] is that over $80 \%$ of the contribution to $J_{\|}$comes from $0<Z<1 \mathrm{R}_{\mathrm{E}}$, implying that there are no significant "sources" (i.e. significant values for the RHS of Eq. 8) above $Z=1 \mathrm{R}_{\mathrm{E}}$. This result can be understood, because in their physical setting only the second term of Eq. 8 is significant. This term has large values in regions with large (non-geodesic) $\boldsymbol{\kappa}$, i.e. very close to the equatorial plane, especially for the over-stretched T89. However, in a more realistic situation with selfconsistent $J_{\|}$, the first term in Eq. 8 will also be significant. For example, Cheng [1995] showed that by integrating just this term for a 3-D computed equilibrium from the equatorial plane to the ionosphere, one obtains values for $J_{\|}$consistent with observations $\left(\sim 1 \mu \mathrm{A} / \mathrm{m}^{2}\right)$. Also, if $J_{\|}$were to mainly originate within $Z=1 \mathrm{R}_{\mathrm{E}}$, then the current within a flux tube would have to stay almost constant as the tube goes from $Z=1 \mathrm{R}_{\mathrm{E}}$ to the ionosphere. A realistic Birkeland current topology probably does not satisfy this requirement - for example, it is evident in the construction of the region 2 currents in T96 [Tsyganenko and Stern, 1996] that the "current tubes" do not coincide with the flux tubes.

\section{A Possible Solution to the Force- Imbalance Problem}

We need to mention first one possible underlying reason of why the fields of an empirical model such as T96 are not in force balance with the pressure. There have been arguments [e.g., Wing and Newell, 2000] that because both field and $P$ observations represent averaged configurations, they ought to nearly satisfy the force balance. However, one recognizes, as Stern [1994a] points out, that while $\mathbf{B}$ in an empirical model might represent the real field with a reasonably good correlation coefficient, first and higher order spatial derivatives of $\mathbf{B}$ are likely to be farther and farther from reality. That T96 indeed gives a rather good prediction of $\mathbf{B}$ is indicated for example by the test performed by Zhou et al. [1997], who only found major discrepancies with observations close to the cusp region. However, it is well known [e.g., Stern, 1994a] that first and higher order derivatives of a function fitted by a least-squares approach are worse approximations of the derivatives of the original function. Thus, we expect $\mathbf{J} \times \mathbf{B}$ as obtained from the model to present a worse correlation against the actual force $\mathbf{J} \times \mathbf{B}$, with $\boldsymbol{\nabla} \cdot(\mathbf{J} \times \mathbf{B})$ being an even more inadequate approximation of its corresponding actual quantity. The most obvious approach would be to build an empirical model that would fit currents instead of magnetic fields. Such a project is not practical in the near future however, due mainly to the lack of synoptic multi-point data.

There is an alternative approach however, namely the adjustment of the model field by computing a 3$\mathrm{D}$ quasi-equilibrium in which force balance is satisfied throughout the domain. Such a study, limited to $15 \mathrm{R}_{\mathrm{E}}$, with $P=P(\psi)$ and using simple boundary flux surface shapes, has been successfully performed in the past using the MAG-3D code [Cheng, 1995]. We have since refined the code to extend the equilibrium to about $25 R_{E}$ and to use observation-based $P$ from both DMSP [Wing and Newell, 1998] and GEOTAIL [e.g., Hori et al., 2000], as well as boundary conditions from the T96 empirical model. Using observed pressure profiles to obtain the magnetic field and not vice-versa also makes physical sense in the plasma sheet, where high values of the plasma $\beta$ indicate that indeed $P$ determines the field, not the other way around. The details of our self-consistent computation will be presented in a future paper. The obtained 3-D configurations have already been used as a background configuration in order to compute field-line resonances [Cheng and Zaharia, 2002], and should prove a useful tool for modelers in other areas where self consistency between magnetospheric fields and plasma is indispensable.

\section{Summary and Conclusions}

Empirical models have been enjoying widespread use in the space physics community, being employed 
in a wide array of applications, ranging from mapping along the field lines to modeling of Birkeland currents, field-line resonance and ballooning instabilities.

Knowledge of the magnetosphere however is not complete if only the average $\mathbf{B}$ is known. The plasma pressure is another crucial factor due to its fundamental effect, especially in high- $\beta$ regions such as the plasma sheet, on the magnetospheric dynamics and the formation of transverse and field-aligned currents. Due to the very limited knowledge of $P$ from in-situ observations, attention has focused to other means of obtaining $P$, one of them being computing a pressure distribution that would be in force balance with the magnetic stresses of an empirical field model.

In this paper we analyze the issue of force balance in the T96 model, with the goal of finding out how well the T96 field structure can support an equilibrium with isotropic $P$. To extract most physics from our treatment, we work in a flux coordinate system in which the empirical field is naturally given in terms of Euler potentials as $\mathbf{B}=\boldsymbol{\nabla} \psi \times \nabla \alpha$ (the many benefits of the Euler representation are discussed in [e.g., Stern, 1994a]).

The previously used method of obtaining a 2dimensional $P$ from an empirical model by simple quadratures is deficient because in an empirical model field configuration the problem is not well posed, as there is no mathematically well-defined $P$ with $\nabla P=$ $\mathbf{J} \times \mathbf{B}$. We present a rigorous way of obtaining $P$ in 2$\mathrm{D}$, by using the decomposition $\mathbf{J} \times \mathbf{B}=\boldsymbol{\nabla} P+\boldsymbol{\nabla} \times \mathbf{G}$ by Helmholtz's theorem, followed by solving a resulting Poisson equation for $P$ in the equatorial plane. The computation is more than 2-D: while $P=P(\psi, \alpha)$ is a function of $\psi$ and $\alpha$ only, components due to the dependence of the operators $\boldsymbol{\nabla}$ and $\nabla^{2}$ on the third coordinate $\chi$ are taken into account as well. The Poisson equation is solved using various boundary conditions on the inner and outer $\psi$ surfaces. Once $P$ is known, $\boldsymbol{\nabla} \times \mathbf{G}$ is also found, and represents a better criterion for judging the departure of a magnetospheric state from equilibrium with isotropic $P$ than previous "isotropy criteria" based on $\boldsymbol{\nabla} \times(\mathbf{J} \times \mathbf{B})$.

We also compute $P$ on the Sun-Earth axis only by integrating $\boldsymbol{\nabla} P=\mathbf{J} \times \mathbf{B}$ (a well-posed problem in 1D). This method has been used in the past for earlier models such as T87 and T89 [e.g., Spence et al., 1987; Kan et al., 1992], however our present calculation is to our knowledge the first such study for T96.

Comparing $P$ obtained via the two methods on the Sun-Earth line, we note that while $P$ obtained from 1-D integration (called $P_{1}$ ) compares relatively well with observations, $P$ from the Poisson equation (denoted by $P_{2}$ ) has significantly higher values throughout the domain. Our results suggest that the T96 model is not compatible with an isotropic pressure in the 3-D domain at any location, including the plasma sheet. The importance of this fact depends on the particular application to which the empirical model is employed, as for some applications its use could be more appropriate than for others. An application where force-balance is absolutely essential is the computation of the field-aligned currents. We perform this computation and we find that using the so-called Vasyliunas relation [Vasyliunas, 1970, 1984] with empirical model fields can lead to spurious currents. As an example we also provide comments on the work of Wing and Newell [2000], who use B from the T89 model in conjunction with $P$ observations to obtain $J_{\|}$ from Vasyliunas relation, but their implicit assumption that force balance is "nearly" satisfied in such a case is not likely to be valid, as we discuss.

We also discuss a possible reason for the lack of force balance in empirical models, which lies in the method by which these models are built, which is by fitting the field from a framework of postulated currents to observed $\mathbf{B}$ data. This approach, while providing $\mathbf{B}$ values that correlate well with observations, could yield less than satisfactory values for key physical quantities computed from the model by differentiation of the field, such as the electric currents.

Finally, we describe a possible solution to the issue of force imbalance in empirical models, namely the "adjustment" of the model field by computing a 3 -D field that satisfies force balance with a pressure gradient, by using boundary conditions from the empirical model and observation-based $P$ profiles. Such a study, using a refined version of the MAG-3D code [Cheng, 1995] with boundary conditions from T96 and pressure from both ionospheric (DMSP) and plasma sheet (GEOTAIL) observations, will be presented in a future paper.

Acknowledgments This work was supported by NASA grant No. W-19512 and DoE contract No. DEAC02-76-CH03073. One of the authors (S.Z.) would like to thank Dr. Jay R. Johnson for numerous discussions and attention to this work. We also thank Dr. N. A. Tsyganenko for making available the computational routines for his empirical models. We acknowledge Dr. D. McCune for the use of the NTCC PSPLINE modules. Finally, we thank C. P. Wang for providing us with their computed proton pressure, 
along with a compilation of pressure observations.

\section{References}

Alexeev, I. I., E. S. Belenkaya, V. V. Kalegaev, Y. I. Feldstein, and A. Grafe, Magnetic storms and magnetotail currents, J. Geophys. Res., 101, 7737, 1996.

Antonova, E. E., and N. Y. Ganyushkina, Reconstruction of large-scale azimuthal pressure gradients in the magnetosphere from field-aligned current data, Geomagn. and Aeron. (English transl.), 35, 610, 1996.

Arfken, G., Mathematical Methods for Physicists, Academic Press, Orlando, FL, 1985.

Baumjohann, W., and G. Paschmann, Determination of the polytropic index in the plasma sheet, Geophys. Res. Lett., 16, 295, 1989.

Baumjohann, W., G. Paschmann, and C. A. Cattell, Average plasma properties in the central plasma sheet, J. Geophys. Res., 94, 6597, 1989.

Birn, J., R. R. Sommer, and K. Schindler, Selfconsistent theory of the quiet magnetotail in three dimensions, J. Geophys. Res., 82, 147, 1977.

Boonsiriseth, A., R. M. Thorne, G. Lu, V. K. Jordanova, M. F. Thomsen, D. M. Ober, and A. J. Ridley, A semiempirical equatorial mapping of AMIE convection electric potentials (MACEP) for the January 10, 1997, magnetic storm, J. Geophys. Res., 106, 12,903, 2001.

Cao, F., and L. C. Lee, Plasma pressure and anisotropy inferred from the Tsyganenko magneticfield model, Ann. Geophys., 12, 286, 1994.

Cheng, C. Z., Magnetospheric equilibrium with anisotropic pressure, J. Geophys. Res., 97, 1497, 1992.

Cheng, C. Z., Three-dimensional magnetospheric equilibrium with isotropic pressure, Geophys. Res. Lett., 22, 2401, 1995.

Cheng, C. Z., and S. Zaharia, Field line resonances in quiet and disturbed time 3-D magnetospheres, 2002, submitted to J. Geophys. Res.

D'haeseleer, W. D., W. N. G. Hitchon, J. D. Callen, and J. L. Shohet, Flux Coordinates and Magnetic Field Structure, Springer-Verlag, Berlin, 1991.
Ding, C., T. W. Hill, and F. R. Toffoletto, Magnetic mapping and Birkeland currents in the ToffolettoHill and Tsyganenko magnetosphere models, $J$. Geophys. Res., 99, 17,343, 1994.

Donovan, E. F., Modeling the magnetic effects of field-aligned currents, J. Geophys. Res., 98, 13,529, 1993.

Elphinstone, R. D., D. Hearn, J. S. Murphree, and L. L. Cogger, Mapping using the Tsyganenko long magnetospheric model and its relationsphip to Viking auroral images, J. Geophys. Res., 96, 1467, 1991.

Hackbusch, W., Elliptic Differential Equations, Theory and Numerical Treatment, Springer-Verlag, Berlin, 1992.

Hall, L. S., and B. McNamara, Three-dimensional equilibrium of the anisotropic, finite-pressure guiding-center plasma, Phys. Fluids, 18, 552, 1975.

Hill, T. W., and G. H. Voigt, Limits on plasma anisotropy in a tail-like magnetic field, Geophys. Res. Lett., 19, 2441, 1992.

Hori, T., K. Maezawa, Y. Saito, and T. Mukai, Average profile of ion flow and convection electric field in the near-Earth plasma sheet, Geophys. Res. Lett., 27, 1623, 2000.

Horton, W., L. Cheung, J. Y. Kim, and T. Tajima, Self-consistent plasma pressure tensors from the Tsyganenko field models, J. Geophys. Res., 98, 17,327, 1993.

Iijima, T., and T. A. Potemra, The amplitude distribution of field-aligned currents at northern high latitudes observed by Triad, J. Geophys. Res., 81, $2165,1976$.

Kan, J. R., W. Sun, and W. Baumjohann, A hybrid equation of state for the quasi-static central plasma sheet, Geophys. Res. Lett., 19, 421, 1992.

Kropotkin, A. P., and A. T. Y. Lui, Quasi-static evolution of the magnetosphere: The substorm growth phase, J. Geophys. Res., 100, 17,231, 1995.

Lu, G., G. L. Siscoe, A. D. Richmond, T. I. Pulkkinen, N. A. Tsyganenko, H. J. Singer, and B. A. Emery, Mapping of the ionospheric field-aligned currents to the equatorial magnetosphere, J. Geophys. Res., 102, 14,467, 1997. 
Lui, A. T. Y., and D. C. Hamilton, Radial profiles of quiet time magnetospheric parameters, J. Geophys. Res., 97, 19,325, 1992.

Lui, A. T. Y., H. E. Spence, and D. P. Stern, Empirical modeling of the quiet time nightside magnetosphere, J. Geophys. Res., 99, 151, 1994.

Mead, G. D., and D. H. Fairfield, A quantitative magnetospheric model devised from spacecraft magnetometer data, J. Geophys. Res., 80, 523, 1975.

Nakamura, M., G. Paschmann, W. Baumjohann, and N. Sckopke, Ion distributions and flows near the neutral sheet, J. Geophys. Res., 96, 5631, 1991.

Nötzel, A., K. Schindler, and J. Birn, On the cause of approximate pressure isotropy in the quiet nearEarth plasma sheet, J. Geophys. Res., 90, 8293, 1985.

Northrop, T., The Adiabatic Motion of Charged Particles, Wiley-Interscience, New York, 1963.

Ober, D. M., N. C. Maynard, W. J. Burke, J. Moen, A. Egeland, P. E. Sandholt, C. J. Farrugia, E. J. Weber, and J. D. Scudder, Mapping prenoon auroral structures to the magnetosphere, J. Geophys. Res., 105, 27,519, 2000.

Olson, W. P., and K. A. Pfitzer, Quantitative model of magnetospheric magnetic-field, J. Geophys. Res., 79, 3739, 1974.

Ostapenko, A. A., and Y. P. Maltsev, Relation of the magnetic field in the magnetosphere to the geomagnetic and solar wind activity, J. Geophys. Res., 102, 17,467, 1997.

Peredo, M., D. P. Stern, and N. A. Tsyganenko, Are existing magnetospheric models excessively stretched ?, J. Geophys. Res., 98, 15,343, 1993.

Peymirat, C., and D. Fontaine, A numerical method to compute Euler potentials for non dipolar magnetic fields, Ann. Geophys., 17, 328, 1999.

Pulkkinen, T. I., D. N. Baker, R. J. Pellinen, J. S. Murphree, and L. A. Frank, Mapping of the auroral oval and individual arcs during substorms, $J$. Geophys. Res., 100, 21,987, 1995.

Rostoker, G., and S. H. Skone, Magnetic flux mapping considerations in the auroral oval and the Earth's magnetotail, J. Geophys. Res., 98, 1377, 1993.
Sergeev, V. A., and M. V. Malkov, Diagnostics of the magnetic configuration of the plasma layer from measurements of energetic electrons above the ionosphere, Geomagn. and Aeron., 28, 549, 1988.

Sergeev, V. A., M. Malkov, and K. Mursula, Testing the isotropic boundary algorithm method to evaluate the magnetic field configuration in the tail, $J$. Geophys. Res., 98, 7609, 1993.

Spence, H. E., and M. G. Kivelson, Contributions of the low-latitude boundary layer to the finite width magnetotail convection model, J. Geophys. Res., 98, 15,487, 1993.

Spence, H. E., M. G. Kivelson, and R. J. Walker, Static magnetic field models consistent with nearly isotropic plasma pressure, Geophys. Res. Lett., 14, 872, 1987.

Spence, H. E., M. G. Kivelson, R. J. Walker, and D. J. McComas, Magnetospheric plasma pressures in the midnight meridian: Observations from 2.5 to $35 \mathrm{R}_{\mathrm{E}}$, J. Geophys. Res., 94, 5264, 1989.

Stern, D., Geomagnetic Euler potentials, J. Geophys. Res., 72, 3995, 1967.

Stern, D., Representation of magnetic fields in space, Rev. Geophys., 14, 199, 1976.

Stern, D. P., The art of mapping the magnetosphere, J. Geophys. Res., 99, 17,169, 1994a.

Stern, D. P., Euler potentials of current-free fields expressed in spherical harmonics, J. Geophys. Res., 99, 2443, 1994b.

Stiles, G. S., J. E. W. Hones, S. J. Bame, and J. R. Asbridge, Plasma sheet pressure anisotropies, $J$. Geophys. Res., 83, 3166, 1978.

Tsyganenko, N. A., Global quantitative models of the geomagnetic field in the cislunar magnetosphere for different disturbance levels, Planet. Space Sci., 35, 1347, 1987.

Tsyganenko, N. A., A magnetospheric field model with a warped tail current sheet, Planet. Space Sci., 37, 5, 1989.

Tsyganenko, N. A., Quantitative models of magnetospheric magnetic field: Methods and results, Space. Sci. Rev., 54, 75, 1990. 
Tsyganenko, N. A., A global analytical representation of the magnetic field produced by the region 2 Birkeland currents and the partial ring current, J. Geophys. Res., 98, 5677, 1993.

Tsyganenko, N. A., Modeling the Earth's magnetospheric magnetic field confined within a realistic magnetopause, J. Geophys. Res., 100, 5599, 1995.

Tsyganenko, N. A., and D. P. Stern, Modeling the global magnetic field of the large-scale Birkeland current systems, J. Geophys. Res., 101, 27,187, 1996.

Vasyliunas, V. M., Mathematical models of magnetospheric convection and its coupling to the ionosphere, in Particles and Fields in the Magnetosphere, ed. B. M. McCormac, D. Reidel, Hingham, $M A$, pp. 60-71, 1970.

Vasyliunas, V. M., Fundamentals of current description, in Magnetospheric Currents, ed. T. A. Potemra, American Geophysical Union, Washington, D. C., p. 63, 1984.

Voigt, G.-H., Field line twist and field-aligned currents in an axisymmetric equilibrium magnetosphere, J. Geophys. Res., 91, 10,995, 1986.

Walker, R. J., and D. J. Southwood, Momentum balance and flux conservation in model magnetospheric magnetic fields, J. Geophys. Res., 87, 7460, 1982.

Wang, C. P., and L. R. Lyons, Modeling the quiet time inner plasma sheet protons, J. Geophys. Res., 106, 6161, 2001.

Weinstock, R., Calculus of Variations, with Applications to Physics and Engineering, Dover, New York, 1974.

Wing, S., and P. T. Newell, Central plasma sheet ion properties as inferred from ionospheric observations, J. Geophys. Res., 103, 6785, 1998.

Wing, S., and P. T. Newell, Quiet time plasma sheet ion pressure contribution to Birkeland currents, $J$. Geophys. Res., 105, 7793, 2000.

Wolf, R. A., The quasi-static (slow-flow) region of the magnetosphere, in Solar-Terrestrial Physics: Principles and Theoretical Foundations: based on the proceedings of the Theory Institute held at Boston College, August 9-26, 1982, pp. 303-368, Kluwer Academic, 1983.
Zhou, X. W., C. T. Russell, G. Le, and N. Tsyganenko, Comparison of observed and model magnetic fields at high altitudes above the polar cap: POLAR initial results, Geophys. Res. Lett., 24, 1451, 1997.

\footnotetext{
This preprint was prepared with the AGU $\mathrm{LAT}_{\mathrm{E}} \mathrm{X}$ macros v3.0. File PPPL report formatted 2002 June 11.

With the extension package ' $\mathrm{AGU}^{++}$', version 1.2 from 1995/01/12
} 


\section{External Distribution}

Plasma Research Laboratory, Australian National University, Australia

Professor I.R. J ones, Flinders University, Australia

Professor J oão Canalle, Instituto de Fisica DEQ/IF - UERJ , Brazil

Mr. Gerson O. Ludwig, Instituto Nacional de Pesquisas, Brazil

Dr. P.H. Sakanaka, Instituto Fisica, Brazil

The Librarian, Culham Laboratory, England

Library, R61, Rutherford Appleton Laboratory, England

Mrs. S.A. Hutchinson, JET Library, England

Professor M.N. Bussac, Ecole Polytechnique, France

Librarian, Max-Planck-Institut für Plasmaphysik, Germany

J olan Moldvai, Reports Library, MTA KFKI-ATKI, Hungary

Dr. P. Kaw, Institute for Plasma Research, India

Ms. P.J . Pathak, Librarian, Insitute for Plasma Research, India

Ms. Clelia De Palo, Associazione EURATOM-ENEA, I taly

Dr. G. Grosso, Instituto di Fisica del Plasma, Italy

Librarian, Naka Fusion Research Establishment, J AERI, J apan

Library, Plasma Physics Laboratory, Kyoto University, J apan

Research Information Center, National Institute for Fusion Science, J apan

Dr. O. Mitarai, Kyushu Tokai University, J apan

Library, Academia Sinica, Institute of Plasma Physics, People's Republic of China

Shih-Tung Tsai, Institute of Physics, Chinese Academy of Sciences, People's Republic of China

Dr. S. Mirnov, TRINITI, Troitsk, Russian Federation, Russia

Dr. V.S. Strelkov, Kurchatov Institute, Russian Federation, Russia

Professor Peter Lukac, Katedra Fyziky Plazmy MFF UK, Mlynska dolina F-2, Komenskeho Univerzita, SK-842 15 Bratislava, Slovakia

Dr. G.S. Lee, Korea Basic Science Institute, South Korea

Mr. Dennis Bruggink, Fusion Library, University of Wisconsin, USA

Institute for Plasma Research, University of Maryland, USA

Librarian, Fusion Energy Division, Oak Ridge National Laboratory, USA

Librarian, Institute of Fusion Studies, University of Texas, USA

Librarian, Magnetic Fusion Program, Lawrence Livermore National Laboratory, USA

Library, General Atomics, USA

Plasma Physics Group, Fusion Energy Research Program, University of California at San Diego, USA

Plasma Physics Library, Columbia University, USA

Alkesh Punjabi, Center for Fusion Research and Training, Hampton University, USA

Dr. W.M. Stacey, Fusion Research Center, Georgia Institute of Technology, USA

Dr. J ohn Willis, U.S. Department of Energy, Office of Fusion Energy Sciences, USA

Mr. Paul H. Wright, Indianapolis, Indiana, USA 
The Princeton Plasma Physics Laboratory is operated by Princeton University under contract with the U.S. Department of Energy.

\author{
Information Services \\ Princeton Plasma Physics Laboratory \\ P.O. Box 451 \\ Princeton, NJ 08543
}

Phone: 609-243-2750

Fax: 609-243-2751

e-mail: pppl_info@pppl.gov

Internet Address: http://www.pppl.gov 